\title{
EPR investigation of electronic excitations in rare gas solids
}

\author{
(Review Article)
}

\author{
R. A. Zhitnikov and Yu. A. Dmitriev \\ A. F. Ioffe Physico-Technical Institute, 26 Politekhnicheskaya, St. Petersburg 194021, Russia \\ E-mail: zhitnikv@mares.ioffe.rssi.ru
}

Received August 14, 1997, revised May 22, 1998

\begin{abstract}
The methods are described for producing unstable paramagnetic excited states in rare gas cryocrystals $\mathrm{Ne}, \mathrm{Ar}, \mathrm{Kr}$, and $\mathrm{Xe}$ through the trapping, in the cryocrystals growing from the gas phase, the products of the gas discharge taking place in the same or other rare gas. The paper presents a technique and results of an observation and investigation of excited states in rare gas cryocrystals with electron paramagnetic resonance (EPR). The discovered unstable paramagnetic centers are interpreted as being local metastable excited $n p^{5}(n+1) s$ atomic-type states in rare gas cryocrystals which are subject to the action of the anisotropic electric field resulted from the crystal surroundings distorted by the center. An account is given of the mechanisms for formation of observed paramagnetic excited states in cryocrystals which arise owing to the excitation energy of the metastable ${ }^{3} \mathrm{P}_{2}$ atoms of $\mathrm{Ne}, \mathrm{Ar}, \mathrm{Kr}, \mathrm{Xe}$ and $\mathrm{He} 2^{3} S_{1}$ and $2^{1} S_{0}$ atoms that form in the discharge in an appropriate gas and trap in the growing cryocrystal.
\end{abstract}

PACS: 76.30.-v

\section{Contents}

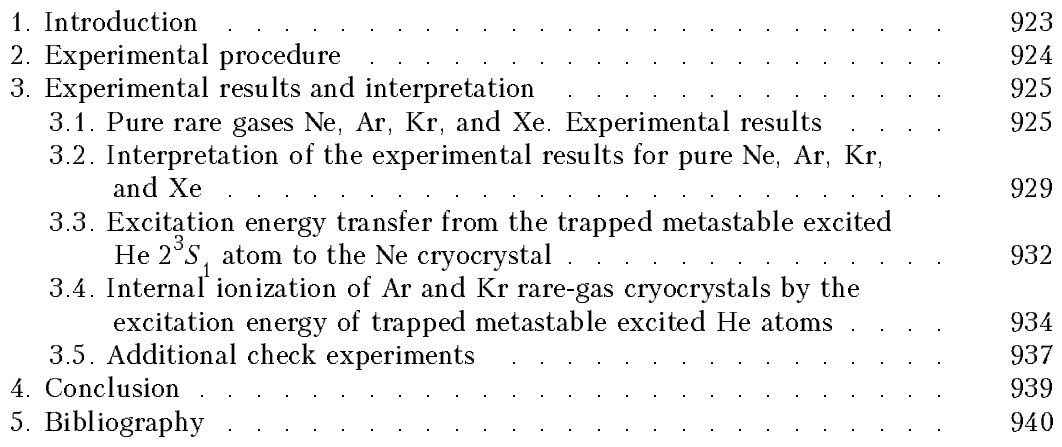

\section{Introduction}

Composed of closed-shell atoms that are bound to each other by the weak Van-der-Waals forces and having, as a rule, the structure of tightly packed face-centered cubic lattice, rare gas cryocrystals, $\mathrm{Ne}, \mathrm{Ar}, \mathrm{Kr}$, and $\mathrm{Xe}$, are the simplest solids in nature. At the same time, the electronic excitations in these crystals are distinguished by abundance of properties and complicated behavior [1-6]. Thus, the free excitons in these simple Van-der-Waals atomic crystals appear to be, contrary to expectations, not mere Frenkel-type excitons but of a more complicated intermediate type that, in many instances, is even closer to Wannier-type excitons. The self-trapping of these excitons, though, can bring into formation at once of even two types of point motionless excitons: molecular two-atom type, $\mathrm{Rg}_{2}^{*}$ (m-STE), and atomic type, Rg* (a-STE), with electron shells and energy levels close to excimer molecules and free excited atoms, respec- 
tively, in the corresponding rare gas. It is also remarkable that all three types of excitons - free and both self-trapped types (molecular and atomic) - are capable of existing in the same crystal simultaneously. This is due to the fact that there exist the activation barriers against the self-trapping of free excitons into both the molecular and atomic types of motionless excitons. The self-trapping processes involve the exciton-phonon interaction and local relaxation deformations of the crystal lattice, which causes in particular the formation of cavities (bubbles) containing excited atoms, Rg*.

These interesting phenomena have attracted considerable attention of many researchers worldwide who undertook extensive studies of electronic excitations in rare gas cryocrystals, in recent decades. The results are detailed in books [1-3] and extensive review articles alike, e.g., [4-6].

It is worth noting that in all studies conducted the excited states in rare gas cryocrystals were obtained by exposure of a cryocrystal grown on a cold surface to ionizing radiation: electrons, ions, $\alpha$-particles and hard enough electromagnetic radiation (VUV or x-rays), while the properties of these electronic excitations in the cryocrystals were examined with the optical spectroscopy methods (luminescence, absorption, reflection, photoelectron emission) [1-6].

In the recent years, researches [7-12] have been performed wherein an entirely new method was used for producing excited states in rare gas crystals. This consists in introducing and trapping the excited products from the rare gas discharge in the cryocrystal of either the same or other rare gas growing from the gas phase on a cold substrate, thereupon the excited states in cryocrystal arising under the action of the trapped particles are studied. In these papers [7-12], as powerful a method as the electron paramagnetic resonance (EPR) is applied for the first time to the study of electronic excitations in rare gas cryocrystals. The present review is mainly devoted to the results of this work. The findings of investigations into electronic excitations in rare gas cryocrystals obtained by conventional methods, that is, with ionizing irradiation of rare gas cryocrystals along with the optical methods of their examination, are available from books and reviews, e.g., [1-6].

\section{Experimental procedure}

The technique employed in [7-12] differs from the known ones as follows. The solid samples under studies are obtained by gas condensation on a thinwalled bottom, used here as the substrate, of a quartz finger filled with liquid helium, this bottom being located at the center of the microwave cavity of an EPR spectrometer. Both the gas discharge and the matrix gas flow passing through a separate inlet tube avoiding the gas discharge, can be cooled down to the liquid nitrogen temperature. The products of the high-frequency gas discharge without intermediate feeding tubes are aimed in a vacuum directly onto the substrate, which prevents them from decay on the walls of the tubes. Thus, the sample is obtained directly in the cavity of the EPR spectrometer, which makes it possible not only to carry out the EPR observation of the sample during the condensation but also to detect and study in the sample short-lived centers that are related to the gas discharge products.

The experimental set-up consists of a 3-cm EPR spectrometer with a microwave cavity cooled by liquid nitrogen vapors, a gas filling and purification system, a high-frequency oscillator for exciting an electrodeless gas discharge, measuring instruments, and pumping facilities [7-12].

Figure 1 shows the main part of the experimental set-up: the microwave cavity of the $3-\mathrm{cm}$ EPR spectrometer, a low-temperature gas discharge device and the substrate cooled by liquid helium, on which the sample is condensed.

Here 1 is the cylindrical $H_{011}$ mode microwave cavity of the $X$-band EPR spectrometer, 2 is the bottom of the quartz finger 3 , filled with liquid helium, and 4 is a waveguide. The bottom 2 of the quartz finger is a low-temperature substrate for the gases being condensed. An electrodeless high-frequency gas discharge is excited in the glass tube 5 with an outlet 6 of $0.2-0.6 \mathrm{~mm}$ diameter. The matrix gas could be supplied to the substrate 2 by a glass tube 7 and further by a quartz tube 8 inserted into the cavity (channel $B$ ). The end of the quartz tube 8 is located close $(3 \mathrm{~mm})$ to the bottom 2 of the quartz finger, which facilitates effective freezing out of the matrix gas.

The whole device presented in Fig. 1 is cooled externally with liquid nitrogen vapor $\left(\mathrm{LN}_{2}\right)$ and its temperature can be varied from 77 to $300 \mathrm{~K}$.

A high-frequency $(14-\mathrm{MHz})$ oscillator is used to maintain the discharge. The high-frequency power is fed through a coaxial cable to the coil 9 wound over the gas discharge tube 5 .

The experimental procedure is as follows. Pure rare gas ( $\mathrm{Ne}, \mathrm{Ar}, \mathrm{Kr}, \mathrm{Xe}, \mathrm{He})$ in channel $A$ was passed through the liquid-nitrogen-vapor-cooled gas discharge tube 5, in which an electrodeless high-frequency discharge was excited. Ground-state rare gas atoms together with gas discharge products 


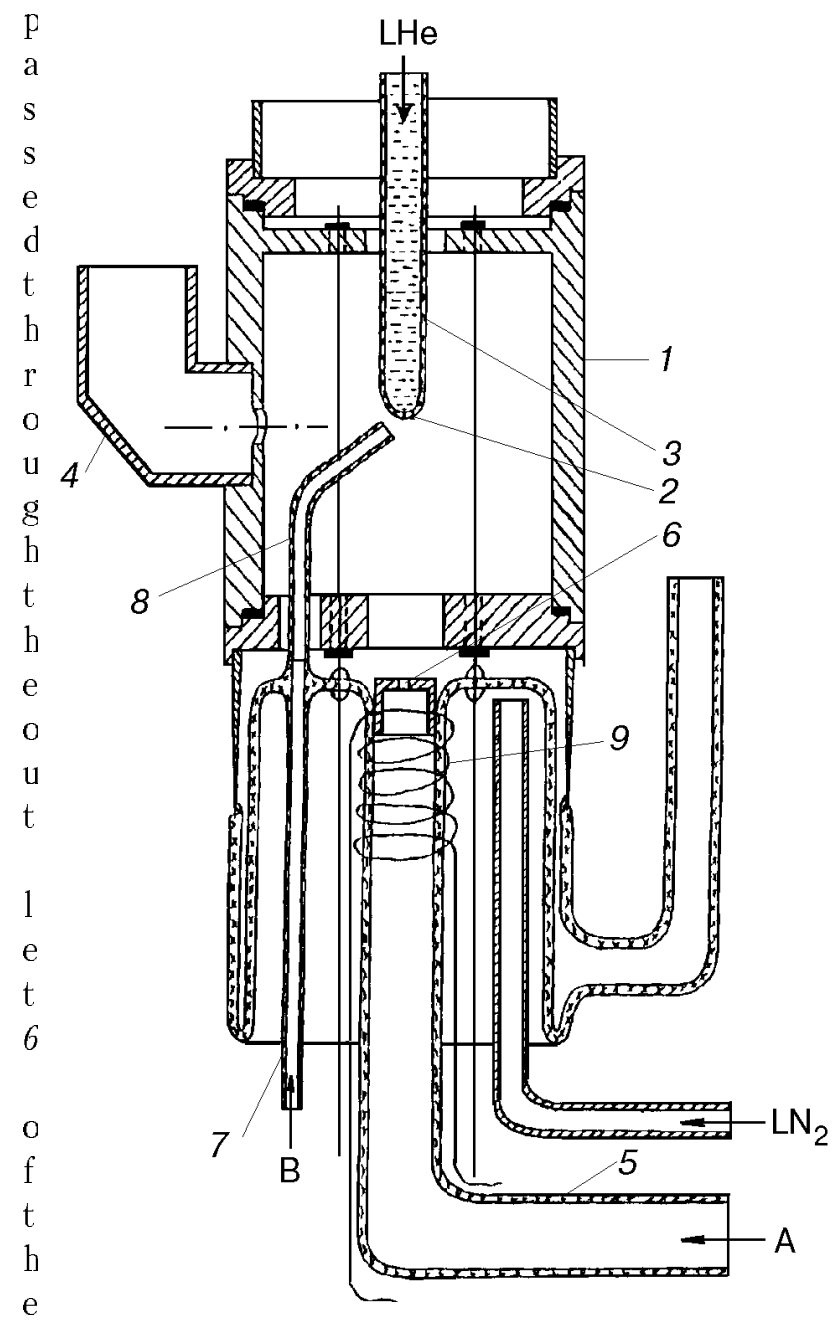

fig. 1. The main part of the experimental set-up including the

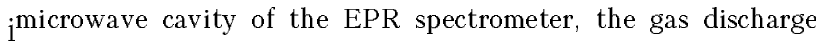
system, the gas inlet tube for matrix gas, and the quartz finger cooled by liquid helium ( $\mathrm{LHe}$ ) with the sample condensing on the bottom of the quartz finger as on a substrate (for notations see the text). $\mathrm{h}$

a

rge tube 5 into the evacuated cavity and reached the bottom 2 of the quartz finger 3 filled with liquid helium at $1.2-4.2 \mathrm{~K}$. The pure additional matrix gas, also cooled by liquid nitrogen vapors $\left(\mathrm{LN}_{2}\right)$, was passed through the tubes 7,8 (channel $B$ ), avoiding the gas discharge, onto the substrate. The EPR spectra of the sample were recorded continuously during its condensation, i.e., growth of the rare gas cryocrystal with gas-discharge products trapped in the sample. The short-lived centers were separated from the stable centers by switching off the discharge, i.e., switching off the voltage on the coil 9 during recording the EPR spectrum. High-purity rare gases were used with the following impurity contents: $0.004 \% \mathrm{Ne}, 0.007 \% \mathrm{Ar}, 0.0009 \% \mathrm{Kr}$, $0.0003 \% \mathrm{Xe}$, and $0.01 \% \mathrm{He}$.

\section{Experimental results and interpretation}

\subsection{Pure rare gases $\mathrm{Ne}, \mathrm{Ar}, \mathrm{Kr}$, and $\mathrm{Xe}$. Experimental results}

In all experiments described in Sec. 3.1, a rare gas $(\mathrm{Ne}, \mathrm{Ar}, \mathrm{Kr}, \mathrm{Xe})$ supplied to the substrate 2 was passed only through the tube 5 (channel $A$ ) with the gas discharge running, while the second channel, $B$, (the tubes 7, 8, Fig. 1) was free from a gas, i.e., closed, and no excess matrix gas was fed through it in the runs of Sec. 3.1.

Figures 2-6 show the EPR spectra of unstable paramagnetic centers which form in a cryocrystal of a pure rare-gas $(\mathrm{Ne}, \mathrm{Ar}, \mathrm{Kr}, \mathrm{Xe})$ being passed through the gas discharge zone, i.e., through the tube 5 only (Fig. 1) that is cooled in these experiments to the liquid-nitrogen temperature, $77 \mathrm{~K}$. These spectra (Figs. 2-6) were observed only during a continuous flow of a rare gas from the tube 5 onto the cold ( $T=1.2-4.2 \mathrm{~K})$ substrate 2 along with the products of the gas discharge taking place in this tube. The EPR signal disappeared at the

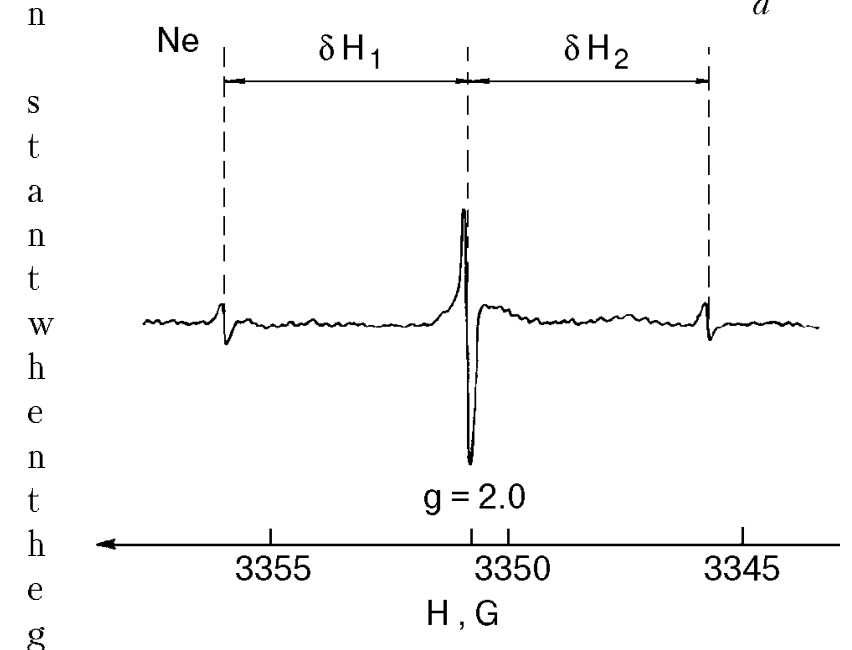

a

$\mathrm{s}$

d

i

$\mathrm{s}$

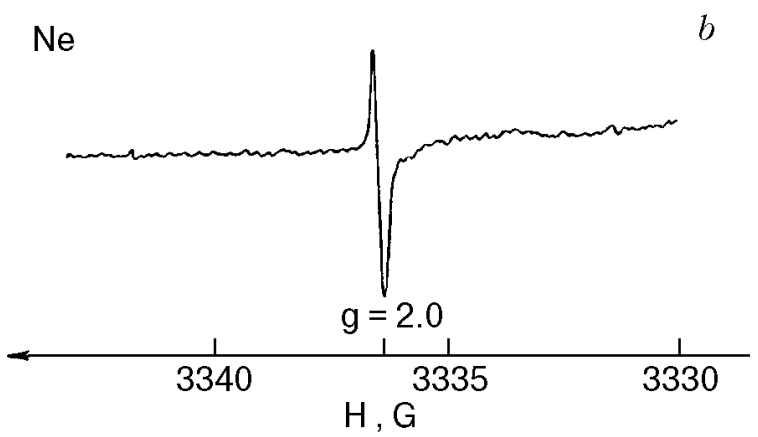

Fig. 2. EPR spectrum of unstable paramagnetic centers in a $\mathrm{W}^{\text {neon }}$ cryocrystal. The gas is let only through the gas discharge tube 5 (Fig. 1). The gas flow rate to the substrate 2 is

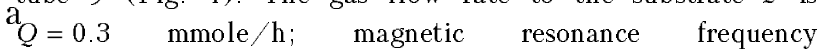
$\mathrm{s}_{f_{\text {res }}}=9379.31 \mathrm{MHz}(a), f_{\text {res }}=9339.00 \mathrm{MHz}(b)$. The side-line tintensity, in Fig. 2,b, reduces as compared to Fig. 2, $a$.

$\mathrm{u}$ 


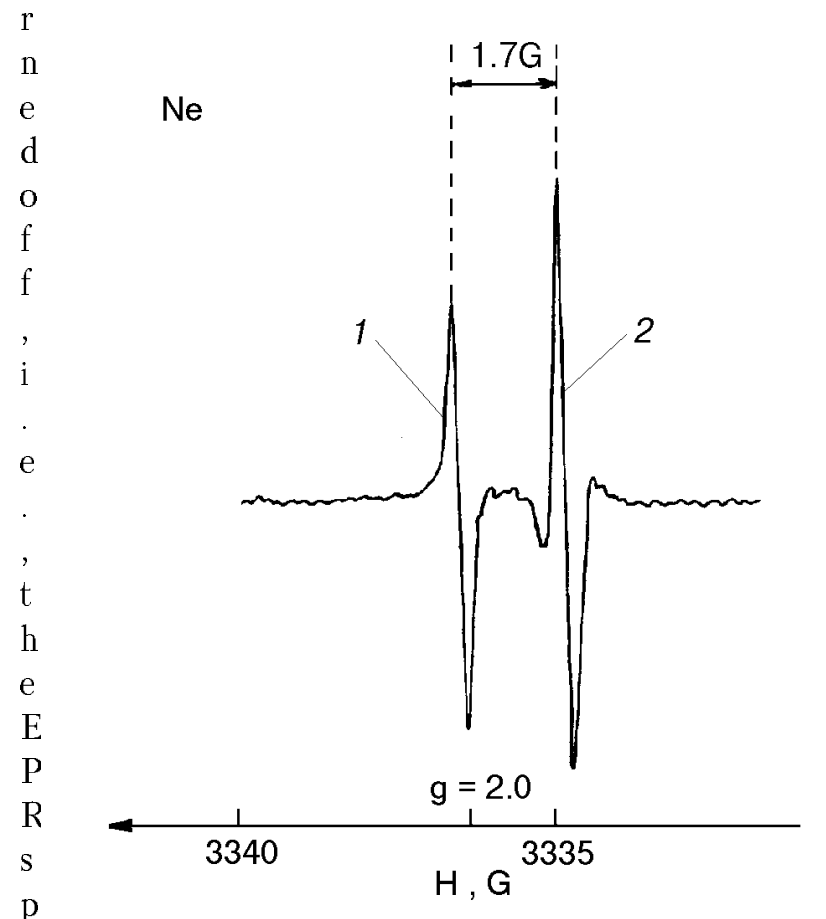

$\mathrm{p}$

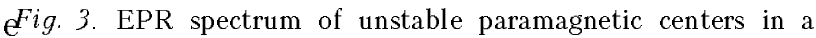
neon cryocrystal with increased gas flow to the substrate $Q=0.7 \mathrm{mmole} / \mathrm{h} ; T=4.2 \mathrm{~K}, f_{\text {res }}=9338.94 \mathrm{MHz}$.

t

rum record was cut off immediately. Thus, the paramagnetic centers responsible for these spectra have short lifetimes. According to estimates based on the time constant of the recording system of the EPR spectrometer, the lifetime of these centers is no more than $0.1 \mathrm{~s}$.

It was found that these paramagnetic centers are localized in a frozen rare gas. Thus, when the discharge is turned on in the tube 5 and in the presence of a rare gas flow from this tube into the cavity, but in the absence of liquid helium in the quartz finger 3 , i.e., when no cryocrystals are formed, the EPR signals indicated above are completely absent. This testifies that the observed short-lived paramagnetic centers are located in the cryocrystals, not in the gas phase. This is also confirmed by an examination of the properties of $\mathrm{t}$ h

e The parameters of EPR spectra of unstable paramagnetic centers in rare gas cryocrystals at low gas flow rate to the substrate $\mathrm{s}(T=4.2 \mathrm{~K})$

\begin{tabular}{|c|c|c|c|c|c|c|c|}
\hline \multirow{2}{*}{$\begin{array}{l}c \\
e\end{array}$} & \multirow{2}{*}{ Rare gas } & \multirow{2}{*}{ No. of runs } & \multirow{2}{*}{$g$-factor } & $\delta H_{1}$ & $\delta H_{2}$ & $\Delta H_{1}^{a}$ & $\Delta H_{2}^{b}$ \\
\hline & & & & \multicolumn{4}{|c|}{ Gauss } \\
\hline $\mathrm{n}$ & $\mathrm{Ne}$ & 7 & $1.99987(12)$ & $5.19(8)$ & $5.24(8)$ & $0.1-0.2$ & $0.1-0.2$ \\
\hline & $\mathrm{Ar}$ & 4 & $1.99986(12)$ & $5.21(8)$ & $5.18(8)$ & $0.1-0.2$ & $0.1-0.2$ \\
\hline t & $\mathrm{Kr}$ & 5 & $1.99986(12)$ & - & - & $0.15-0.25$ & - \\
\hline $\mathrm{e}$ & $\mathrm{Xe}$ & 4 & $1.99983(12)$ & - & - & $0.2-0.3$ & - \\
\hline
\end{tabular}

\footnotetext{
$a$ - here $\Delta H_{1}$ is the width of the central line; $b$ - here $\Delta H_{1}$ is the widths of the side lines.
}

s, which are discussed below.

The EPR spectra, obtained at comparatively low gas flow rate through the gas discharge (no more than $0.5 \mathrm{mmole} / \mathrm{h}$ ), were found, for the cryocrystals of pure neon and argon, to be completely identical both in appearance (i.e., as in Fig. 2) and in all quantitative characteristics (also see Ref. 8). Nor were any differences observed between the spectra obtained at the substrate temperatures $T=$ $=1.2$ and $4.2 \mathrm{~K}$. The parameters of these spectra coincide completely within the measurement error for neon and argon cryocrystals and are given in Table. The $g$-factor quoted there refers to the middle (brightest) line in Fig. 2. The other designations are clear from this figure.

The relative strengths of the center and satellite lines in the spectra shown in Fig. 2 change from run to run both in neon and argon, from the maximum strength of the satellite lines to the point they are completely unobservable. The variability of the central line to side line intensity ratio indicates that the side doublet and the center line belong to different paramagnetic centers.

As the gas flow through the discharge tube increases, and when the flow to the substrate 2 exceeds $0.7 \mathrm{mmole} / \mathrm{h}$, new lines appear in the spectra of the short-lived centers in the neon and argon cryocrystals, and in the process the lines shown in Fig. 2 are preserved. Figure 3 gives the middle region of the EPR spectrum for neon for an increased gas flow rate. Two closely spaced lines are seen, with line 1 coinciding with the center line in Fig. 2 and having a $g$-factor equal to that given in Table. The spacing between these lines amounts to $1.7 \mathrm{G}$. Figure 4, $a$ shows the EPR spectrum for $\mathrm{Ar}$ also for an increased gas flow rate. Here line 1 coincides with the center line in Fig. 2, and the new line is separated from it by $1.6 \mathrm{G}$. Thus, as the gas flow grows, the same changes may occur in the EPR spectra of short-lived centers for neon and argon, as follows from the similarity of the spectra in Figs. 3 and 4,a. In some experiments, however, for the

Table 


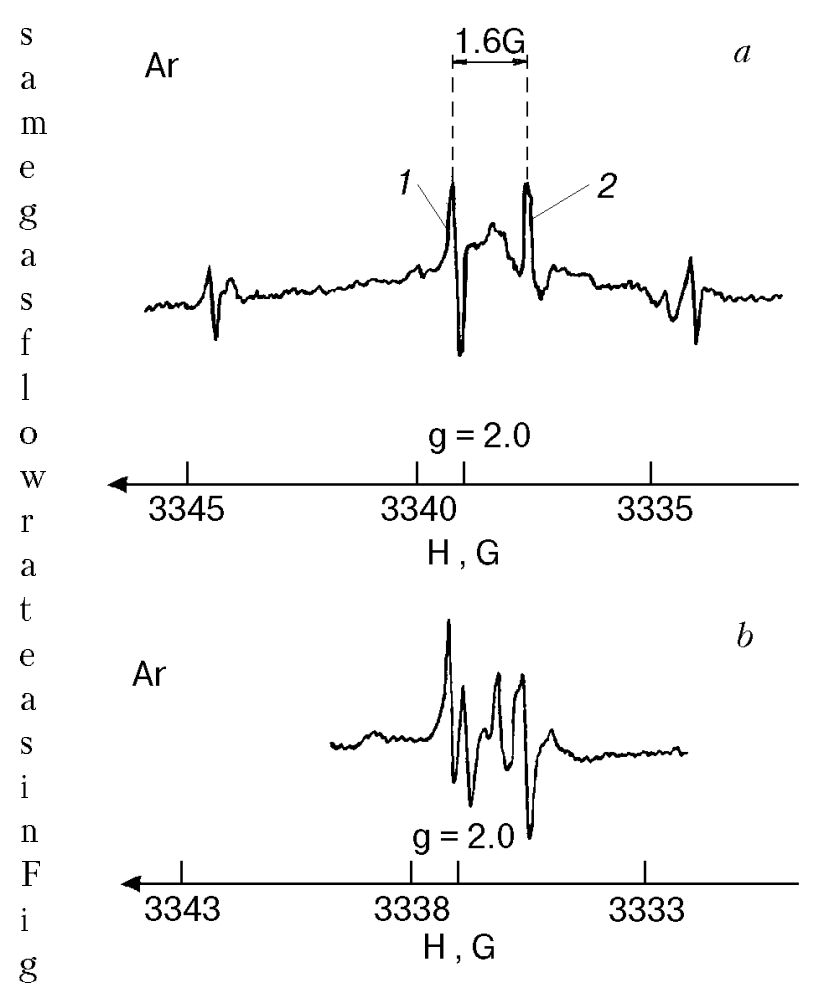

Fig. 4. EPR spectrum of unstable paramagnetic centers in an argon cryocrystal with increased gas flow to the substrate at $T=4.2 \mathrm{~K}$ and $Q=2.2 \mathrm{mmole} / \mathrm{h}, f_{\text {res }}=9346.60 \mathrm{MHz}(a)$; ${ }^{\prime} f_{\text {res }}=9340.93 \mathrm{MHz}(b)$.

two more lines appeared between the two center lines shown in this figure, and all four lines had comparable strengths (see Fig. 4,b).

The EPR spectra of $\mathrm{Kr}$ and Xe cryocrystals obtained at the same conditions as for the spectrum in Fig. 2, i.e., when a rare-gas flow rate is low, consist each of one line (see Figs. 5, $a$ and 6,a), which lines, as indicated by Table, are identical in $g$-factor, within the accuracy of the experiment, to one another and to the middle lines in the spectra of $\mathrm{Ne}$ and Ar. These lines are also close in linewidths for all the four rare gases. One can, thus, believe that the principal unstable paramagnetic center is of a common nature to all the four rare-gas cryocrystals produced under similar conditions, i.e., at modest flow rate through the gas discharge.

In EPR spectra of $\mathrm{Kr}$ and Xe cryocrystals no side lines provided by the other unstable center were observed, as it was in Ne and Ar (Figs. 2 and 4,a). As indicated above, sometimes these side lines could not be observed in $\mathrm{Ne}$ and Ar also. At present one cannot be sure whether the centers that give side lines are absent in $\mathrm{Kr}$ and Xe cryocrystals or whether these centers in the experiments were too scarce to be spectroscopically observable.

Shown in Figs. 5, $b$ and 6,b, are the experimental results for $\mathrm{Kr}$ and $\mathrm{Xe}$ obtained with the gas dis- charge in tube 5 at room temperature, i.e., without liquid-nitrogen cooling the tube. These spectra differ from those in Figs. 5, $a$ and 6, $a$, although they are equally short-lived, i.e., disappear immediately when the discharge is turned off. In the spectrum of $\mathrm{Xe}$, the number, location and intensity of lines changed from one experiment to the next. But they were close to one another within the confines of the spectrum of Fig. 6,b, were located around $g=2.0$, and the entire spectrum disappeared immediately once the discharge was turned off.

It should be noted that the excess lines arising with the increased gas flow rate vary in their characteristics ( $g$-factors, location, linewidths) with the kind of the rare gas used (Figs. 3, 4, a, 4,b, $5, b, 6, b)$, and reveal no such a similarity they do in the spectra of the rare gases with the low gas flow rate, presented in Figs. 2, 5, $a, 6, a$, and Table.

All the excess lines occurring with an increased gas flow rate onto the substrate are unstable just as the lines presented in Fig. 2 and Table are, and disappear along with the principal lines when the d

s

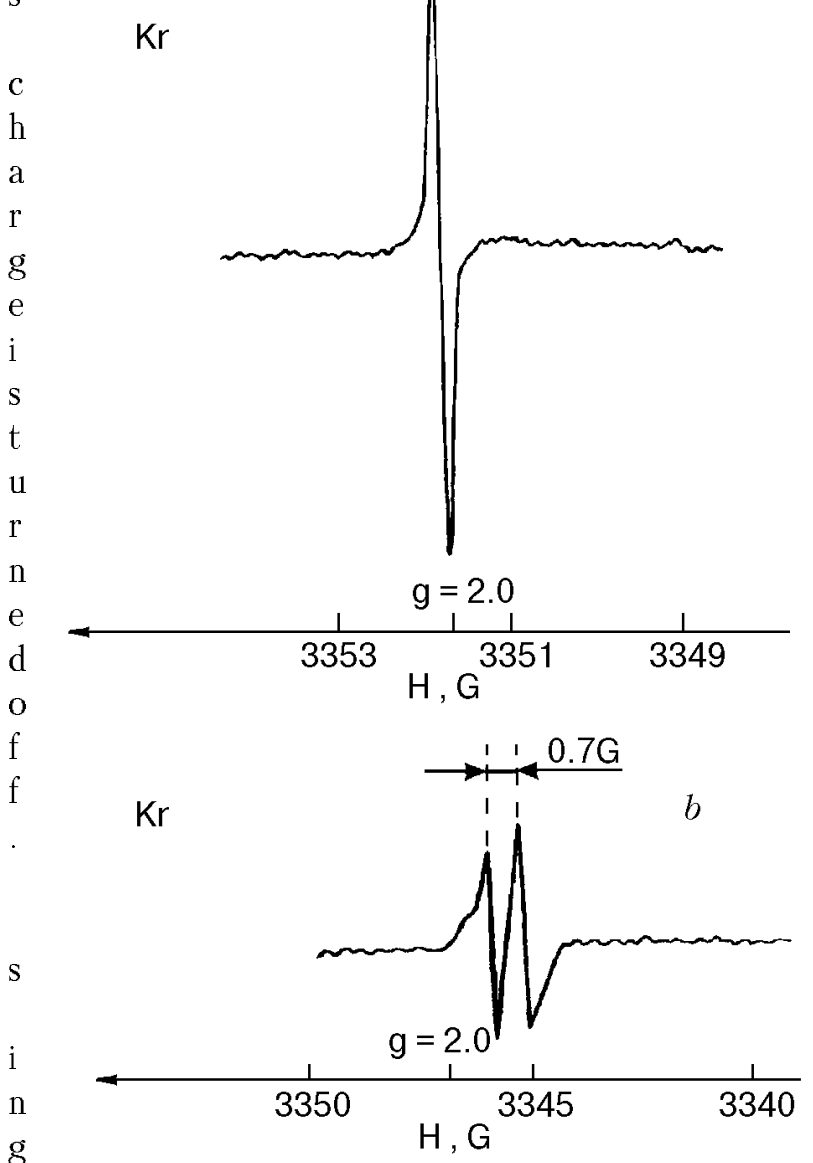

tFig. 5. EPR spectrum of unstable paramagnetic centers in hapton cryocrystal at $T=4.2 \mathrm{~K}, Q=0.2 \mathrm{mmole} / \mathrm{h} ; f_{\text {res }}=$ $\mathrm{e}=9382.04 \mathrm{MHz}(a) ; Q=0.5 \mathrm{mmole} / \mathrm{h}, f_{\text {res }}=9368.63 \mathrm{MHz}(b)$.

E 


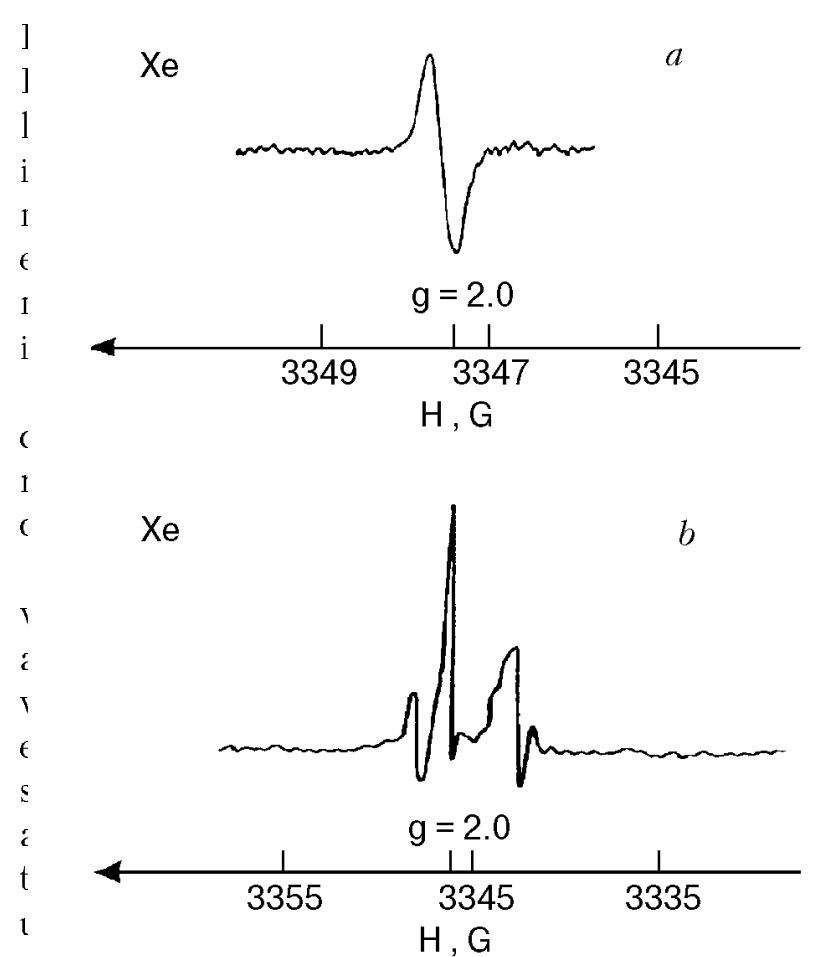

${ }_{\mathrm{r}}$ Fig. 6. EPR spectrum of unstable paramagnetic centers in a xenon cryocrystal at $T=4.2 \mathrm{~K}, Q=0.04 \mathrm{mmole} / \mathrm{h}, f_{\text {res }}=$ $=9370.15 \mathrm{MHz}(a) ; Q=0.6 \mathrm{mmole} / \mathrm{h}, f_{\text {res }}=9366.92 \mathrm{MHz}(b)$.

$\mathrm{t}$

ion method, the lower boundary for the lifetime $\tau$ of the unstable paramagnetic centers was determined in all cryocrystals, $\mathrm{Ne}, \mathrm{Ar}, \mathrm{Kr}$, and Xe [8]. These lifetime values are found to be in the interval $10^{-1} \mathrm{~s} \geq \tau \geq 10^{-4} \mathrm{~s}$ for certain of these centers, and in the interval $10^{-1} \mathrm{~s} \geq \tau \geq 10^{-2} \mathrm{~s}$ for others [8].

\subsection{Interpretation of the experimental results for pure $\mathrm{Ne}, \mathrm{Ar}, \mathrm{Kr}$, and $\mathrm{Xe}$}

To elucidate the nature of the observed shortlived paramagnetic centers, it is necessary to consider the products of the discharge in a rare gas that may enter the cryocrystal of this gas being condensed, as well as the possible action of the gas discharge on the cryocrystal already frozen onto the substrate, and to compare the results of this examination with the properties and characteristics of the EPR spectra shown in Figs. 2-6 and in the Table.

In order to reach the cold substrate 2 on which the sample is condensed, the gas-discharge products must travel a distance $\sim 3 \mathrm{~cm}$ through vacuum. If they are rare-gas atoms their thermal velocities are of order $10^{4} \mathrm{~cm} / \mathrm{s}$, i.e., only particles with a lifetime $\geq 10^{-4} \mathrm{~s}$ can reach the substrate. The only neutral products of the discharge in the rare gas which satisfy this condition are excited metastable atoms of this gas in the lower ${ }^{3} P_{2}$ and ${ }^{3} P_{0}$ states (for free ${ }^{3} P_{2}^{0}$ atoms, the lifetimes have the values
[13] $\tau(\mathrm{Ne})=20 \mathrm{~s}, \tau(\mathrm{Ar})=60 \mathrm{~s}, \tau(\mathrm{Kr})=85 \mathrm{~s}, \tau(\mathrm{Xe})=$ $150 \mathrm{~s}$, and for ${ }^{3} P_{0}^{0}$ atoms, the lifetimes range from $400 \mathrm{~s}$ for neon to $0.08 \mathrm{~s}$ for xenon). The entry of charged particles, i.e., electrons and ions, from the gas discharge into a growing cryocrystal of rare gas, as well as bombardment by these particles of the cryocrystal already formed, is impossible, since observation of the EPR spectra of short-lived centers is carried out as the cryocrystal condenses. Therefore, the strong magnetic field of the EPR spectrometer, $H_{0}=3300 \mathrm{G}$, is turned on all the time; it is transverse to the flow of these charged particles, deflects them to the side, and prevents them from reaching the substrate 2 , i.e., the sample. Thus, for 1 -keV electrons in a 3300-Gauss magnetic field, the orbit radius is $0.32 \mathrm{~mm}$, and still considerably less for thermal-energy ions $\left(r_{i}<0.1 \mathrm{~mm}\right)$, i.e., all charged particles from the gas discharge (both electrons and ions) are locked up firmly with magnetic field in the gas discharge zone 9, and cannot reach the substrate 2 .

Finally, the solid cryocrystal of a rare gas already formed on the substrate, as well as the gas being condensed, can be both subjected to irradiation by light from the gas discharge, including VUV. The action of the light from the discharge in a rare gas on the cryocrystal of this gas or gas at the instant of condensation can produce only the shortlived ${ }^{3} P_{1}$ and ${ }^{1} P_{1}$ atoms. These excited atoms cannot cause the formation of the observed EPR spectra, since the radiative lifetimes of the ${ }^{3} P_{1}$ and ${ }^{1} P_{1}$ atoms are too short: for all rare gases, they are of order $10^{-8}-10^{-9} \mathrm{~s}$. Such lifetimes should result in the EPR linewidths no less than 5-50 G, whereas the observed spectra have linewidths of the order of $0.1 \mathrm{G}$ (see Table and Fig. 2); this requires a lifetime of the paramagnetic center of the order of $5 \cdot 10^{-7} \mathrm{~s}$. At the same time, it is known that the lifetime, for example, of the ${ }^{3} P_{2}$ atoms of neon in a cryocrystal of this gas is no less than $6 \cdot 10^{-4} \mathrm{~s}$ [14]. As indicated above, the lifetimes of the unstable centers observed in the present study $\tau \geq 10^{-4}-10^{-2} \mathrm{~s}$. Therefore, the paramagnetic centers based on the ${ }^{3} P_{2}$ rare gas atoms trapped in cryocrystals should satisfy the requirement of a fairly small EPR linewidth, consistent with that observed experimentally. However, these ${ }^{3} P_{2}$ atoms, like ${ }^{3} P_{0}$ atoms, cannot be practically formed under action of radiation from the gas discharge since the radiation transitions ${ }^{1} S_{0} \rightarrow{ }^{3} P_{2},{ }^{3} P_{0}$ to these metastable states from the ground state ${ }^{1} S_{0}$ are forbidden. Hence, the radiation from the gas discharge cannot be responsible for the formation of the paramagnetic centers producing the observed EPR spectra. 
Thus, the observed centers (Figs. 2-6) can only be due to the entry into the rare gas cryocrystal of atoms of this gas in the ${ }^{3} P_{2}$ state directly from the gas discharge. Atoms in the ${ }^{3} P_{0}$ state, which can also reach the crystal from the discharge, should be excluded from the consideration, since they are diamagnetic and cannot form paramagnetic centers in the cryocrystal.

Hence, it must be taken as proved that the cause of the formation of the observed short-lived paramagnetic centers is the trapping, in a growing rare-gas cryocrystal, of excited atoms of this gas in the metastable ${ }^{3} P_{2}$ state. This atom is paramagnetic and, as was noted above, can have a sufficiently long lifetime in the cryocrystal to provide the experimentally observed small EPR linewidths, $\Delta H_{1,2} \sim 10^{-1} \mathrm{G}$.

However, a significant difficulty arises here. This is because all the lines in the observed EPR spectra have $g$-factors that are very close to $g=2.0$ (Table), whereas the ${ }^{3} P_{2}$ state has the $g$-factor of 1.5 , and its lines should be at entirely different magnetic fields (i.e., for $g=1.5, H_{\text {res }}=4400 \mathrm{G}$, and for $g=2.0, H_{\text {res }}=3300 \mathrm{G}$, as is the case experimentally). It was found experimentally that the EPR spectra are completely absent in magnetic fields close to $4400 \mathrm{G}$. To explain this discrepancy the following was suggested [7-12]. Presumably, the trapped ${ }^{3} P_{2}$ rare gas atom is stabilized in the cryocrystal of the same gas in such a manner that the effective crystal electric field acting on it is anisotropic and of low enough symmetry. The action of the crystal electric field of the tetragonal symmetry on the energy levels of the $P$-state atom is shown in Fig. 7 (see Ref. 15, § 11.5, Fig. 11.2). It is seen from the figure that a crystal field of low enough symmetry lifts the degeneracy of the $P$-state energy levels and causes, in the case of the tetragonal symmetry field, for example, the splitting $\delta$ between $P_{z}\left(m_{L}=0\right)$ and $P_{x}, P_{y}\left(m_{L}= \pm 1\right)$ states. If the splitting $\delta$ is sufficiently large, then at liquid helium temperature $(1.2$ to $4.2 \mathrm{~K})$ almost only the lower $m_{L}=0$ level will be populated, which has the $g$-factor defined, in the tetragonal field, as (see Ref. $15, \S 11.6) g_{z z}=g_{\|}=g_{e}, g_{x x}=g_{\perp}=g_{e}-2 \lambda / \delta$. Here $g_{e}$ is the spin $g$-factor of the free electron, the exact value being $g_{e}=2.00232, \lambda$ is the fine structure constant for the free ${ }^{3} P$ atom of the rare gas at hand, which appears in the spin-orbit interaction hamiltonian, $H=\lambda \mathbf{L} \mathbf{S}$. The constant $\lambda$ therewith is evaluated from the equation $\lambda=\Delta_{\text {so }} / S(L+1)=$ $=\Delta_{s o} / 3$, here $\Delta_{s o}$ is the total splitting of the fine structure for the ${ }^{3} P$ atom considered, $L$ and $S$ are correspondingly its total orbital and spin moments; for the ${ }^{3} P$-state, $L=S=1$. This treatment is strict- ly correct for the atom in a state with the normal, i.e., $[L, S]$-coupling. However, it is known that the rare gas atoms in the state with $n p^{5}(n+1) s$ electron configuration show the departure from the $[L, S]$ coupling, this actually being intermediate between $[L, S]$ and $[j, j]$, that is $\left[J^{\prime}, j\right]$ - coupling. The Ne $2 p^{5} 3 s^{3} P$-atom therewith still follows the interval rule and definitely shows $[L, S]$-coupling. Because of this, the calculation of $\lambda$ is quite justified for $\mathrm{Ne}$ with the formula $\lambda=\Delta_{s o} / 3$. Based on the total spin-orbit splitting for the $\mathrm{Ne} 2 p^{5} 3 s^{3} P$-atom, $\Delta_{\text {so }}=0.097 \mathrm{eV}$ [13], one gets $\lambda(\mathrm{Ne})=0.032 \mathrm{eV}$. Starting with Ar, the considerable departure from the $[L, S]$-coupling for the $n p^{5}(n+1) s$ rare-gas atom is beginning to emerge, which rises for $\mathrm{Kr}$ and $\mathrm{Xe}$ as the atomic mass increases. However, this expression, $\lambda=\Delta_{s o} / 3$, is likely can be used in estimating expected $g$-factors for these atoms in a crystalline field; here, taken from Ref. $13, \Delta_{s o}$ is also the total splitting for three lower levels of the $n p^{5}(n+1) s$ configuration of the free rare-gas atoms. In that event $\lambda(\mathrm{Ar})=0.06 \mathrm{eV}, \lambda(\mathrm{Kr})=0.24 \mathrm{eV}, \lambda(\mathrm{Xe})=0.42 \mathrm{eV}$. If the splitting caused by an anisotropic crystal field is large, so that $\delta>>2 \lambda$ for all the cryocrystals (i.e., $\mathrm{Ne}, \mathrm{Ar}, \mathrm{Kr}$, and $\mathrm{Xe}$ ), then from the above expressions it follows that $g_{\|}=g_{\perp}=g_{e}=2.0$, i.e., the g-factors of the $n p^{5}(n+1) s{ }^{3} P_{2}$-atoms trapped in the corresponding cryocrystals turn isotropic, equal to 2.0 and are close in magnitude (i.e., $g=g_{\text {isotr }}=2.0$ ), which agrees with and explains the result of the experiments (see Fig. 2 and Table).

The spectrum consisting of two side lines in Figs. 2 and $4, a$ can be explained as follows. If an atom in the ${ }^{3} P_{2}$ state is localized in the position in the crystal where the effective electric crystal field has axial symmetry, the state with $m_{L}=0$ in Fig. 7 splits in a zero magnetic field into two spin sublevels with $m_{s}= \pm 1$ and 0 (see Ref. 15, § 10.3). For this case, the levels of the atom in the magnetic field and the EPR transitions are shown in Fig. 8. Thus, the transitions $m_{s}=0 \leftrightarrow m_{s}=+1$ and $m_{s}=$ $=0 \leftrightarrow m_{s}=-1$ will move apart along the magnetic field to give rise to two noncoincident EPR lines $\mathrm{s}$

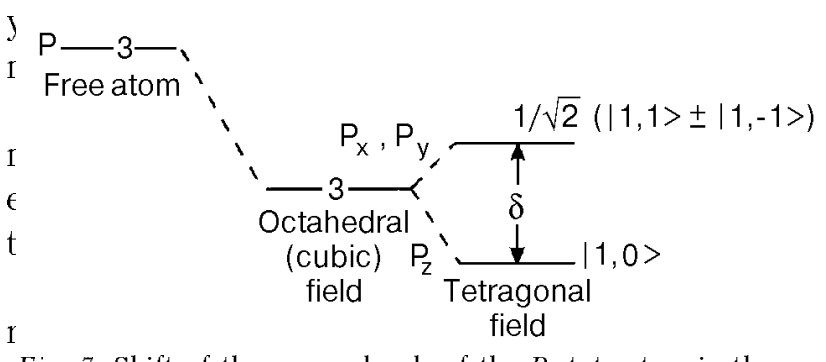
iFig. 7. Shift of the energy levels of the $P$-state atom in the octahedral crystal field followed by the splitting in the tetragonal field. 


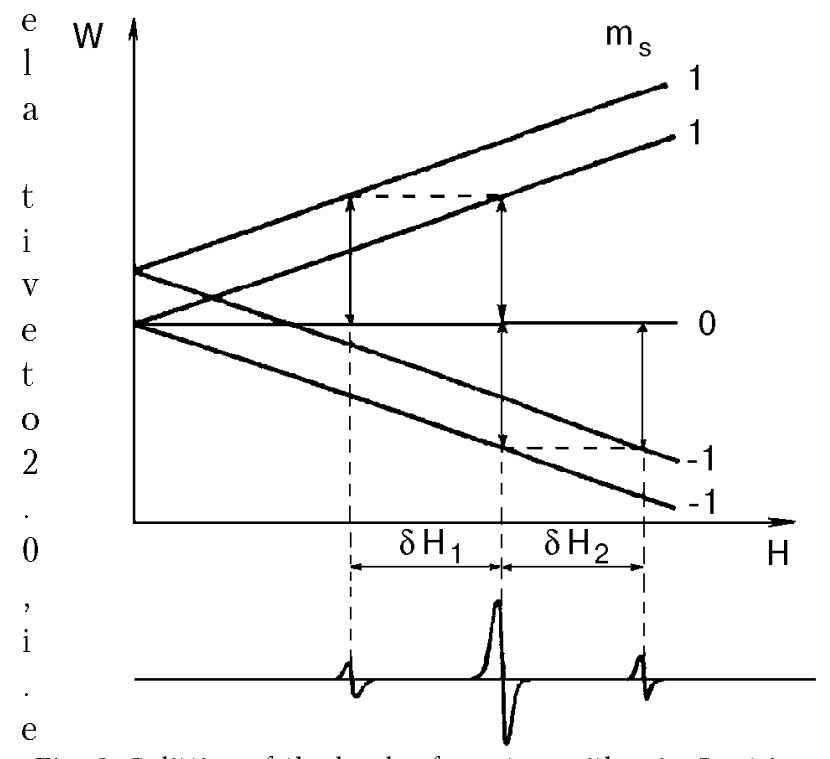

Fig. 8. Splitting of the levels of an atom with spin $S=1$ in an axial electric crystal field and appearance of side lines in the $\mathrm{t}^{\mathrm{E} P R}$ spectrum.

$\mathrm{h}$

e side lines observed in the experiment. From this figure, taking the values for $\delta H_{1}=\delta H_{2}=\delta H_{1,2}=5.2 \mathrm{G}$ from Table, one can determine the splitting $3 / 2 D$ of the levels $m_{s}= \pm 1$ and 0 for $\mathrm{Ne}$ and $\mathrm{Ar}$ in a zero magnetic field: $3 / 2 D=\delta H_{1,2} g \beta / h=2.8 \cdot 10^{6} \cdot \delta H_{1,2} \mathrm{~Hz}=$ $=14.6 \mathrm{MHz}$. The fraction of ${ }^{3} P_{2}$ atoms trapped at the sites with the crystal field of that kind is apparently small and can vary from run to run, which accounts for the variability of the side lines in Fig. 2.

One may suppose that most of the ${ }^{3} P_{2}$ rare gas atoms are trapped in the substitutional position of the face centered cubic (FCC) crystal of the rare gas and give rise to the intensive central line in the EPR spectra in Fig. 2, and a lesser part of the ${ }^{3} P_{2}$ atoms are trapped into octahedral interstitial sites of this crystal to give the side lines. The fraction of the latter atoms is likely to vary from experiment to experiment depending on hard-to-control variations in experimental conditions.

Note that in an undistorted FCC crystal, these trapping sites have a crystal electric field of very high (cubic) symmetry, so that splitting of the $P$-state cannot result, as evident from Fig. 7. A low-symmetry (anisotropic) crystal field providing the observed EPR spectra of trapped atoms with $g=2.0$ appears presumably due to crystal lattice distortions caused by the outer $s$ shell of this $n p^{5}(n+1) s{ }^{3} P_{2}$ atom. Distortion of a rare gas FCC crystal lattice by a rare gas $P$ atom trapped therein as well as local rearrangement of this lattice were considered in Refs. 3,16,17. In the above works, it is shown that the origin of the anisotropic crystal field which act on the trapped $n p^{5}(n+1) s P$-atom and, hence, is able to provide a splitting $\delta$, Fig. 7 , is as follows. The $n p^{5}(n+1) s$ Rydberg state of a rare gas atom has a radius much greater than the ground $n p^{6}$-state of a lattice atom has, thus the $(n+1) s$-electron of the trapped atom gives rise to outward displacement of the surrounding lattice atoms, resulting in a considerable rearranging of the nearest surroundings of the Rydberg excited atom. Such a rearranging of this surroundings breaks the cubic symmetry and produces a local electric field of low symmetry acting on the $n p^{5}(n+1) s P$-atom. This moving apart of the closed-shell $n p^{6}$ atoms of the crystal lattice that surround the trapped excited $n p^{5}(n+1) s$ atom is triggered by the repulsion forces that are due to the requirement, according to the Pauli's principle, for the s-electron wave function of the trapped excited atom be orthogonal to the wave functions of the surrounding lattice atoms. Such a shift of the lattice atoms results in the formation of a cavity around the trapped excited atom, putting it in a so-called bubble [1-3]. A thorough theoretical study of this issue have been undertaken and the estimation of the bubble radius of $4 \AA$ for the $2 p^{5} 3 s$ excitation in Ne cryocrystal was obtained. In Ref. 17, a theoretical treatment is given of formation around the $2 p^{5} 3 s$ excitation in the Ne crystal of, first, bubbles with symmetric elastic moving apart the surrounding $\mathrm{Ne}$ atoms of the lattice, second, bubbles that has induced the plastic distortion of the surroundings consisting in the production in the nearest lattice surroundings of defects in the Frenkel pair form, that is, vacancies and interstitial atoms. It is stated in the paper, that the bubbles with plastic distortion have lower energy than the pure elastic bubbles. In the Ne cryocrystal, the bubbles with two vacancies in the first coordination sphere and interstitial Ne atoms in the next more distant spheres are deduced [17] to have the lowest energy. Regular bubbles with only elastic distortion around the $n p^{5}(n+1) s$ state are of cubic symmetry [17]. Though the plastic deformations with vacancies arising in the first coordination sphere around the $n p^{5}(n+1) s$ excitation, cause a lower symmetry distortion of the surroundings [17], thus creating an anisotropic electric crystal field acting on the trapped excited atom. It is worth noting that this local electric field acting on the $n p^{5}(n+1) s$-atom should be not only quite anisotropic but strong as well. The latter quality is due to the fact that it is not the mere Van-der-Waals interaction that contributes to the local electric field (this interaction therewith should be much 
greater for the excited $n p^{5}(n+1) s P$-state, resulting from its large polarizability, than that one which is characteristic of two rare gas atoms, $n p^{6}$ groundstate of the crystal lattice including), but, for Rydberg atoms, the much stronger chemical bonding between a trapped $n p^{5}(n+1) s$ excited atom and a $n p^{6}$ lattice atom should also appear. This is precisely the strong chemical bonding that gives rise, under proper conditions, to diatomic excimer molecules of rare gases: $\operatorname{Rg}\left({ }^{1} S_{0}\right)+\operatorname{Rg}\left({ }^{3} P_{2}\right) \rightarrow \operatorname{Rg}_{2}^{*}\left({ }^{3} \Sigma_{u}^{+}\right)$. This chemical bonding, of course, should contribute in some measure to the interaction between the $n p^{5}(n+1) s$-atom trapped in rare gas crystal and its anisotropic crystal surroundings. This may provide, for the atom, a great enough splitting $\delta$ to meet the condition $\delta>>2 \lambda$. In so doing, not only does the magnitude of $\lambda$ increases, as is evident from the foregoing, with increasing mass of the rare-gas atom in the range between $\mathrm{Ne}$ and $\mathrm{Xe}$, but the interaction energy between the trapped $n p^{5}(n+1) s$ atom and crystal surroundings also increases including both Van-der-Waals (because the polarizability of the ground-state and metastable ${ }^{3} P_{2}$ atoms alike enhances) interaction and chemical bonding. This, probably, provides the condition $\delta>>2 \lambda$ for the atoms of all rare gases, resulting in $g=2.0$ and closing together of $g$-factors of $\mathrm{Ne}, \mathrm{Ar}, \mathrm{Kr}$ and $\mathrm{Xe}$ with low gas flow rates to the substrate (see Table).

The process of distortion of the lattice by the $P$-atom may proceed through metastable intermediate variants of the configuration of the neighborhood with certain delays in these configurations. Each of these configurations of the immediate neighborhood should be associated with a different symmetry of the crystal electric field acting on the ${ }^{3} P_{2}$ atom. This probably accounts for the appearance, as the experimental conditions change (increasing the gas flow rate onto the substrate 2 or the enhancement of discharge temperature), of new lines in the EPR spectra for $\mathrm{Ne}, \mathrm{Ar}, \mathrm{Kr}$, and $\mathrm{Xe}$ (Figs. 3-6), because of variation in $\delta$ and consequently $g$-factors of the EPR lines. Indeed, an increase in the flow rate of a rare gas or in its temperature may result in heating of the sample, growth of the size of its crystallites, and appearance of trapped ${ }^{3} P_{2}$ atoms for which the process of distortion of the surroundings now takes place far from the surface of the sample, in the interior of the crystals and at a higher temperature. This may cause a change in the conditions of local rearrangement of the lattice around certain groups of ${ }^{3} P_{2}$ atoms and may give rise to the additional lines in the spectra of Figs. 3-6.
It is possible that a contribution to the destruction of the cubic crystal environment of the ${ }^{3} P_{2}$ atoms trapped in rare gas crystal in the described experiments comes also from the dynamic JahnTeller effect [18].

It is noteworthy that the large lifetimes $\tau \geq 10^{-2}-10^{-4} \mathrm{~s}$ found in this work (see Sec. 3.1) eliminate the possibility of explaining the observed EPR spectra as being due to rare gas excimer molecules, $\operatorname{Rg}_{2}^{*}\left({ }^{3} \Sigma_{u}^{+}\right)$, trapped in cryocrystals. Indeed, the lifetimes, $\tau\left[\operatorname{Rg}_{2}^{*}\left({ }^{3} \Sigma_{u}^{+}\right)\right]$, of these molecules in the rare gas cryocrystals are shown [6] to be $\tau\left(\mathrm{Ne}_{2}^{*}\right)=$ $=5 \cdot 10^{-6} \mathrm{~s}, \quad \tau\left(\mathrm{Ar}_{2}^{*}\right)=1.2 \cdot 10^{-6} \mathrm{~s}, \quad \tau\left(\mathrm{Kr}_{2}^{*}\right)=3.2 \cdot 10^{-6} \mathrm{~s}$, $\tau\left(\mathrm{Xe}_{2}^{*}\right)=9 \cdot 10^{-6} \mathrm{~s}$, respectively, i.e., much shorter than the lifetimes $\tau$ found in this work for paramagnetic centers for which $\tau \geq 10^{-2}-10^{-4} \mathrm{~s}$.

Thus, it was demonstrated that the unstable paramagnetic centers observed are due to the rare gas $n p^{5}(n+1) s^{3} P_{2}$ atoms which, once trapped from the gas discharge in a growing cryocrystal, become localized in it, deform and rearrange their surroundings and are subject to the action of an anisotropic electric field of this distorted surroundings. As a result, the local paramagnetic metastable $n p^{5}(n+1) s$ atomic-type excited states observed by EPR and described above arise in the cryocrystals.

It should be noted that the self-trapped excitons of $n p^{5}(n+1) s$ atomic type are known to form efficiently under the action of ionizing radiation in $\mathrm{Ne}$ cryocrystals and with somewhat lesser efficiency in Ar cryocrystal. In irradiated cryocrystals of the heavy rare gases, $\mathrm{Kr}$ and $\mathrm{Xe}$, though, only selftrapped excitons of molecular type, m-STE $\mathrm{Rg}_{2}^{*}$, are observed, while self-trapped excitons of atomic type, a-STE, are not found. The explanation is as follows [1-6]: in $\mathrm{Kr}$ and Xe cryocrystals, the activation barriers against self-trapping into a-STE are very high, while with self-trapping into $\mathrm{m}$-STE these are significantly lower. With $\mathrm{Ne}$ and $\mathrm{Ar}$, the a-STE self-trapping barriers are well below these barriers in $\mathrm{Kr}$ and $\mathrm{Xe}$, which makes possible the self-trapping into a-STE in Ne and Ar. A possibility for the local excited $n p^{5}(n+1) s$ atomic-type states to form in $\mathrm{Kr}$ and Xe cryocrystals in the experiments described above is likely to be due to the method of producing the excited atomic-type states which differs fundamentally from the free exciton self-trapping in these cryocrystals. The point is that, in experiments described above, the excited $n p^{5}(n+1) s^{3} P_{2}$ rare gas atoms are inserted directly into a rare gas cryocrystal growing from the gas phase and trapped in the cryocrystal. This process does not involve an overcoming the self-trapping activation barrier and in this regard the localization 


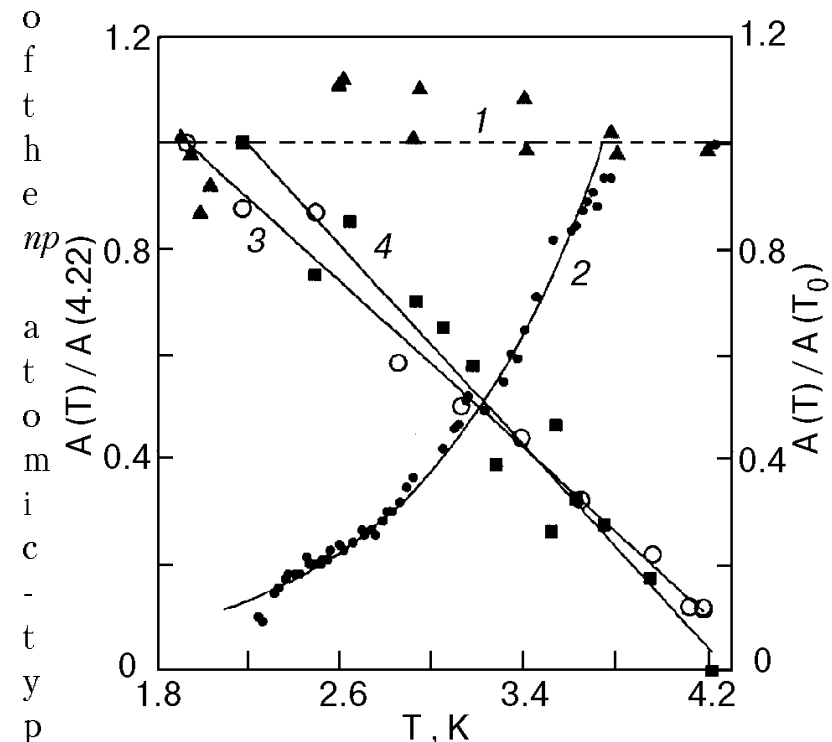

eFig. 9. Temperature dependences of the EPR signal amplitude,

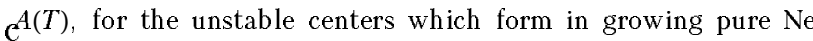
cryocrystal as it traps $\mathrm{Ne}$ gas discharge products (line 1 , triangles), and for the centers which form in growing $\mathrm{Ne}$ (line 2, Đilled circles), Ar (line 3, open circles) and Kr (line 4, squares) cryocrystals as they trap He gas discharge products. $\mathrm{t}$

$\mathrm{e}$

rs should proceed in $\mathrm{Kr}$ and Xe cryocrystals as unhindered as it does in $\mathrm{Ne}$ and $\mathrm{Ar}$.

Figure 9 , line 1 , shows the temperature dependence of the signal amplitude obtained for the central line of the spectrum for Ne in Fig. 2. It can be seen, that the number of observed unstable centers that form in the cryocrystal is independent of the temperature, i.e., the equilibrium number of these centers is invariant to the temperature with the gas flow rate and discharge condition remaining constant. Precisely the same independence from temperature was observed for the side lines of the spectrum for $\mathrm{Ne}$ in Fig. 2, and for all lines of the spectra presented in Figs. 3-6. It must be added to the above, that the experiments show the lifetimes $\tau$ of all unstable paramagnetic centers investigated in the present work to be independent of temperature. Thus, with both the lifetimes of the paramagnetic centers and the flow rate of $n p^{5}(n+1) s^{3} P_{2}$ atoms from the gas discharge, the independence from temperature of the equilibrium number of these centers (Fig. 9, line 1) agrees well with the mechanism proposed: the observed unstable centers are formed through the trapping and localizing in a growing cryocrystal the rare-gas ${ }^{3} P_{2}$ atoms from the gas discharge.

\subsection{Excitation energy transfer from the trapped metastable excited $\mathrm{He} 2^{3} S_{1}$ atom to the $\mathrm{Ne}$ cryocrystal}

In this study, investigations have been carried out where pure $\mathrm{He}$ was passed through the gas discharge (channel $A$ in Fig. 1) while pure $\mathrm{Ne}$ was fed to the substrate through a separate inlet tube (channel $B$ ) avoiding the gas discharge active part. A new effect has been found: the formation in a neon cryocrystal of local metastable excited states arising as helium gas discharge products are trapped in the growing cryocrystal $[9,10]$.

The EPR spectrum of the neon cryocrystal trapping He gas discharge products are shown in Fig. 10. These spectra were observed during the neon condensation and disappeared at the instant when the discharge was turned off. For simplicity the term «edge-side lines» shall be used to denote the outer broad side lines in Fig. 10, and the term «side lines» to refer to the inner narrow side lines.

There is absolute coincidence between both line positions and linewidths of the three middle lines in the $\mathrm{He}-\mathrm{Ne}$ spectrum (Fig. 10) and the corresponding pure Ne lines shown in Fig. 2. Indeed, the $g$-factor and linewidth of the central line of the $\mathrm{He}-\mathrm{Ne}$ spectrum recorded here appear to be 1.99986(12) and 0.15(3) G, respectively, and exactly the same as those for the pure Ne cryocrystal, 1.99987(12) and 0.10(3) G. The side-line linewidths as well as separations between central and side lines are also the same; for the pure Ne these $\mathrm{a}$
$\mathrm{r}$
$\mathrm{e}$
$\mathrm{s}$
$\mathrm{u}$
$\mathrm{m}$
$\mathrm{r}$
$\mathrm{i}$
$\mathrm{z}$
$\mathrm{e}$
$\mathrm{d}$

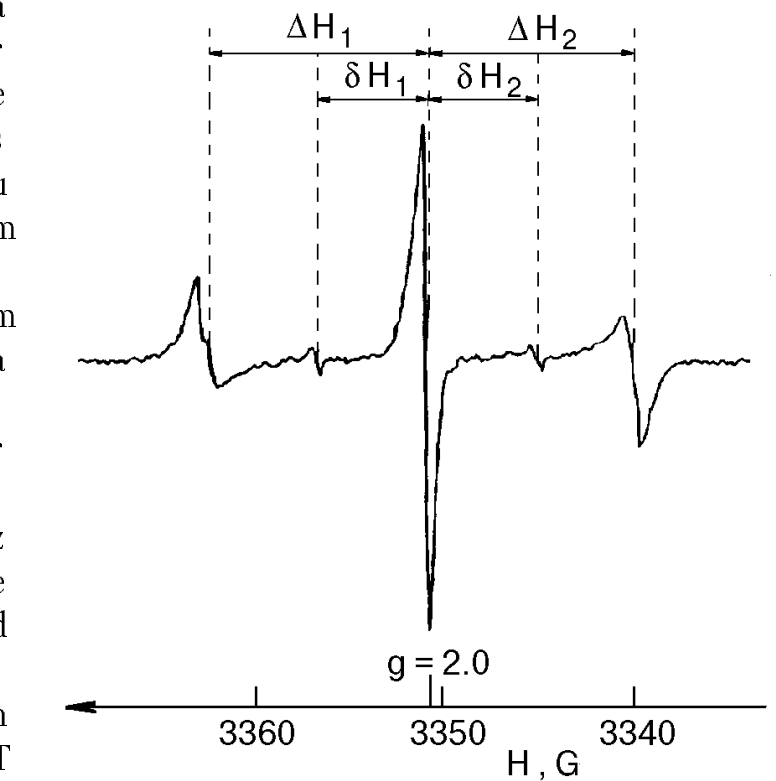

aFig. 10. EPR spectrum of unstable paramagnetic centers in a neon cryocrystal, which arise as a result of a He gas disbharge product trapped in the growing $\mathrm{Ne}$ cryocrystal; $f_{\text {res }}=9381 \mathrm{MHz}$.

$\mathrm{e}$ 
However, there is a significant difference between the spectra of the Ne cryocrystal obtained by condensation of the pure Ne passed through the discharge active part, and the spectra of solid $\mathrm{Ne}$ trapping $\mathrm{He}$ gas discharge products. The latter contains a pair of additional strong edge-side lines of unstable centers, located around central line and separated from it by $\Delta H_{1}$ and $\Delta H_{2}$, for the highfield and low-field lines, respectively. The separation in magnetic field between each edge-side line and the central line is a factor of about 2 above the distances between each of the two weaker inner side lines and central line.

The fact that three middle lines of the He-Ne spectrum recorded here (Fig. 10) are clearly those of the pure Ne spectrum means that these spectra are due to centers of the same type and permits an explanation of the nature of the He-Ne spectrum in the same way as have been done in Sec. 3.2 for pure Ne. Thus, the centers being discussed in the present section can be interpreted also as being atomic-type excited $2 p^{5} 3 s$ states which become localized in the FCC Ne crystal lattice, shift the nearest matrix atoms outwards, rearrange their surroundings and are subjected to the action of the anisotropic electric field of this environment which, being rearranged, is no longer of cubic symmetry.

The nature of the strong edge-side lines, which occur only when a neon cryocrystal is trapping the He gas discharge products, will be discussed below. It is believed that these are also related to the $2 p^{5} 3 s$ centers, but with the surroundings distorted in a different way.

While the nature of the neon cryocrystal excited states recorded here is believed to be clear, the mechanism of their production is to be determined. Indeed, in the experiments on pure rare gases (Sec. 3.1), the excited $\mathrm{Ne}^{3} \mathrm{P}_{2}$ atoms (as well as $\mathrm{Ar}, \mathrm{Kr}$ and Xe atoms), which are trapped in the growing cryocrystal of the same kind and rearrange the nearby crystal lattice, form directly in the gas discharge, whereas in the present study the gas flows aimed onto the substrate contain no excited $\mathrm{Ne}$ atoms at all, including ${ }^{3} \mathrm{P}_{2}$ atoms. Apart from ground-state $\mathrm{He}$ atoms, the only incoming $\mathrm{He}$ gas discharge products at the growing $\mathrm{Ne}$ cryocrystal are the metastable $\mathrm{He} 2{ }^{3} S_{1}$ and $\mathrm{He} 2{ }^{1} S_{0}$ atoms. Other excited helium atoms have too short life times to reach the substrate from the gas discharge. Charged particles from the discharge can also be excluded from consideration, since they are deflected from the substrate by the strong magnetic field of the EPR spectrometer (see Sec. 3.2).
Thus, the unstable paramagnetic centers observed here, which are probably the atomic-type $2 p^{5} 3 s$ excited states of the neon cryocrystal, originate from the excitation energy of incoming metastable He atoms from the gas discharge.

Figure 9 , line 2, shows a plot of $A(T) / A\left(T_{1}\right)$ as a function of $T$, where $A(T)$ is the EPR signal intensity of the central line of the He-Ne spectrum (see Fig. 10) and $T_{1}=4.22 \mathrm{~K}$. In these experiments, the substrate temperature or, correspondingly, the temperature of the sample growing on the substrate 2 (Fig. 1) is lowered by the pumping of liquid helium vapor. The sample temperature is detected by measuring the gas pressure in the liquid helium bath.

It can be seen from Fig. 9, line 2, that the experimental dependence $A(T) / A\left(T_{1}\right)$ can be fairly well approximated by $A(T) / A\left(T_{1}\right)=\exp (-E / k T)$ with the «activation energy» $E=12 \mathrm{~K}=0.001 \mathrm{eV}$. Using either increasing or decreasing sample temperature, many such experimental dependences in the temperature range $2.2-4.2 \mathrm{~K}$ were recorded. Taking into account the statistical scattering of the experimental data, the «activation energy» can be estimated as $E=12(6) \mathrm{K}=0.0010(5) \mathrm{eV}$.

The lifetime $\tau$ of the $2 p^{5} 3 s^{3} P_{2}$-state in the neon cryocrystal was found to be independent of temperature.

Our explanation of the effect found in the present section is as follows $[9,10]$.

The energy level of the He $2^{3} S_{1}$ atom appears to be located in the $X$-excitation energy band of the $\mathrm{Ne}$ cryocrystal, near the top of this band. This permits one to suppose that there exists an effective energy transfer from the metastable He $2^{3} S_{1}$ atom to the neon cryocrystal $X$-exciton band. It is believed that the most probable way of producing $2 p^{5} 3 s$ atomictype states in neon cryocrystal is the quasi-resonance excitation energy transfer from the metastable $\mathrm{He} 2^{3} S_{1}$ atom to the neon cryocrystal $X$-exciton band followed by the self-trapping of the $X$-exciton into the $2 p^{5} 3 p$ ten-level state which then decays to the lower $2 p^{5} 3 s$ four-level excited state whose lowest ${ }^{3} P_{2}$ level is just that observed in the experiment. It is well known from the optical investigations that the self-trapped $X$-exciton is actually the $2 p^{5} 3 p$ atomic-type state decaying to the $2 p^{5} 3 s$ state with luminescence in the visible region $[9,10]$.

The excitation energy $(19.82 \mathrm{eV})$ transfer from the He $2^{3} S_{1}$ atom to the neon cryocrystal $X$-exciton band should be a quasi-resonance process, since the center of the band is at $19.2 \mathrm{eV}$ while the band half-width is about $0.6 \mathrm{eV}$. Thus, the energy transfer from the He $2^{3} S_{1}$ atom is not of a completely resonant nature. The fact that the «activation en- 
ergy», i.e., the «defect of resonance» (or energy misfit), of the energy-transfer process is so small $(E=0.001 \mathrm{eV})$ can be explained as being due to the broad wings of the $X$-exciton band. Temperature dependence of the probability of such an energy transfer seems likely to be determined by the neon cryocrystal phonon spectrum and the shape of the high-energy wing of the band in the region close to $19.8 \mathrm{eV}[10]$.

The above mechanism of production of the Ne $2 p^{5} 3 s$ centers, which explains their existence as being the result of trapping the metastable He $2^{3} S_{1}$ atoms in the growing neon cryocrystal, enables an explanation of the additional edge-side lines separated by $\Delta H_{1}$ and $\Delta H_{2}$ from the central line in the EPR spectrum of the $2 p^{5} 3 s$ centers shown in Fig. 10. The origin of these lines is presumably similar to that of the weak side lines centered around the central line at the distances $\delta H_{1}$ and $\delta H_{2}$, which has already been discussed in Sec. 3.2. Each of these pairs of lines possibly results from the action of an axially symmetric anisotropic crystalline electric field on the $2 p^{5} 3 s$ atomic-type center, which is due to the outward shift, the distortion and the rearrangement of the $2 p^{5} 3 s$-center nearest surroundings in the neon cryocrystal. When a ${ }^{3} P_{2}$ neon atom is trapped in the neon cryocrystal directly from the neon discharge, as it has a large radius, it causes the neighboring matrix atoms to shift outwards, thus producing a rearrangement of its nearest surroundings. This is the reason why the axial field occurs, which gives rise to the weak side lines displaced from the central line by $\delta H_{1}$ and $\delta H_{2}$. On the other hand, when metastable He atoms are trapped in the neon cryocrystal there may be additional deformation of the Ne lattice in the vicinity of the $2 p^{5} 3 s$ atomic-type state which are produced according to the mechanism described earlier in this section. This deformation can be provided by the energy produced during the $X$-exciton self-trapping and by the fact that the radius of the $2 p^{5} 3 p$-state, which is intermediate in the process of $2 p^{5} 3 s$-state formation in neon cryocrystal, is even larger than that of the ${ }^{3} P_{2} \mathrm{Ne}$ atom. This additional lattice deformation occurring around some of the local excitations formed gives rise to new paramagnetic $2 p^{5} 3 s$-centers yielding the strong edge-side lines centered around the central line at the distances $\Delta H_{1}$ and $\Delta H_{2}$ from this (Fig.10)
3.4. Internal ionization of $\mathrm{Ar}$ and $\mathrm{Kr}$ rare-gas cryocrystals by the excitation energy of trapped metastable excited He atoms

All energy levels of $\mathrm{Ar}$ and $\mathrm{Kr}$ atoms lie far below the lowest excited He level. Therefore, the excitation energy cannot be transferred from the trapped metastable excited He atom to the exciton bands of $\mathrm{Ar}$ and $\mathrm{Kr}$ cryocrystals through either resonance or quasi-resonance processes which is the case for the Ne cryocrystal, as described in the previous section.

In the present section, detection and investigation are reported of a new phenomenon: internal ionization of the rare-gas cryocrystals $(\mathrm{Ar}$ and $\mathrm{Kr}$ ) by $\mathrm{He}$ gas discharge excited products (the metastable $2^{3} S_{1}$ and $2^{1} S_{0}$ He atoms) trapped in the growing cryocrystals followed by the formation in these cryocrystals of the local metastable excited $n p^{5}(n+1) s$ atomic-type states.

The experimental set-up used here is shown in Fig. 1. The matrix gas ( $\mathrm{Ar}, \mathrm{Kr})$ flow passes through a separate inlet tube 7-8 (channel $B$ ) avoiding the gas discharge and is cooled to liquid-nitrogen temperature. The high-frequency $(14 \mathrm{MHz})$ strong continuous He gas discharge in channel $A$ is used.

Figures 11, 12 show the EPR spectra of the growing argon and krypton cryocrystals trapping He gas discharge products. These spectra were observed only during the $\mathrm{Ar}$ or $\mathrm{Kr}$ gas condensation and disappeared at the instant when the He discharge was turned off. On the other hand, no EPR spectra were observed in experiments where $\mathrm{He}$ flow was passed through the tube 5 with the radiofrequency discharge running but with no $\mathrm{Ar}$ or $\mathrm{Kr}$ gas being fed onto the substrate through the inlet tube. It was found that the He gas discharge used cannot provide any EPR spectra before the Ar $(\mathrm{Kr})$ condensation as well as after the $\mathrm{Ar}(\mathrm{Kr})$ flow is turned off despite a solid $\mathrm{Ar}(\mathrm{Kr})$ layer condensed on the substrate 2 in a long time run. This is also true of the experiments with He gas discharge and $\mathrm{Ne}$ cryocrystals which are described in Sec. 3.3. These results are strong demonstration that the unstable centers observed in Refs. 7-12 cannot be due to the action of the light from the gas discharge on the rare gas cryocrystals.

The fact that the lines of the spectra recorded here are clearly those of the pure $\mathrm{Ar}$ and $\mathrm{Kr}$ spectra (Figs. 5, $a$ and 6,a) means that the present spectra and the pure-gas spectra are due to centers of the same type and permits one to give an explanation of the nature of the present centers in the same way as has been done previously (Sec. 3.2). Namely, the centers observed here can be interpreted as being 


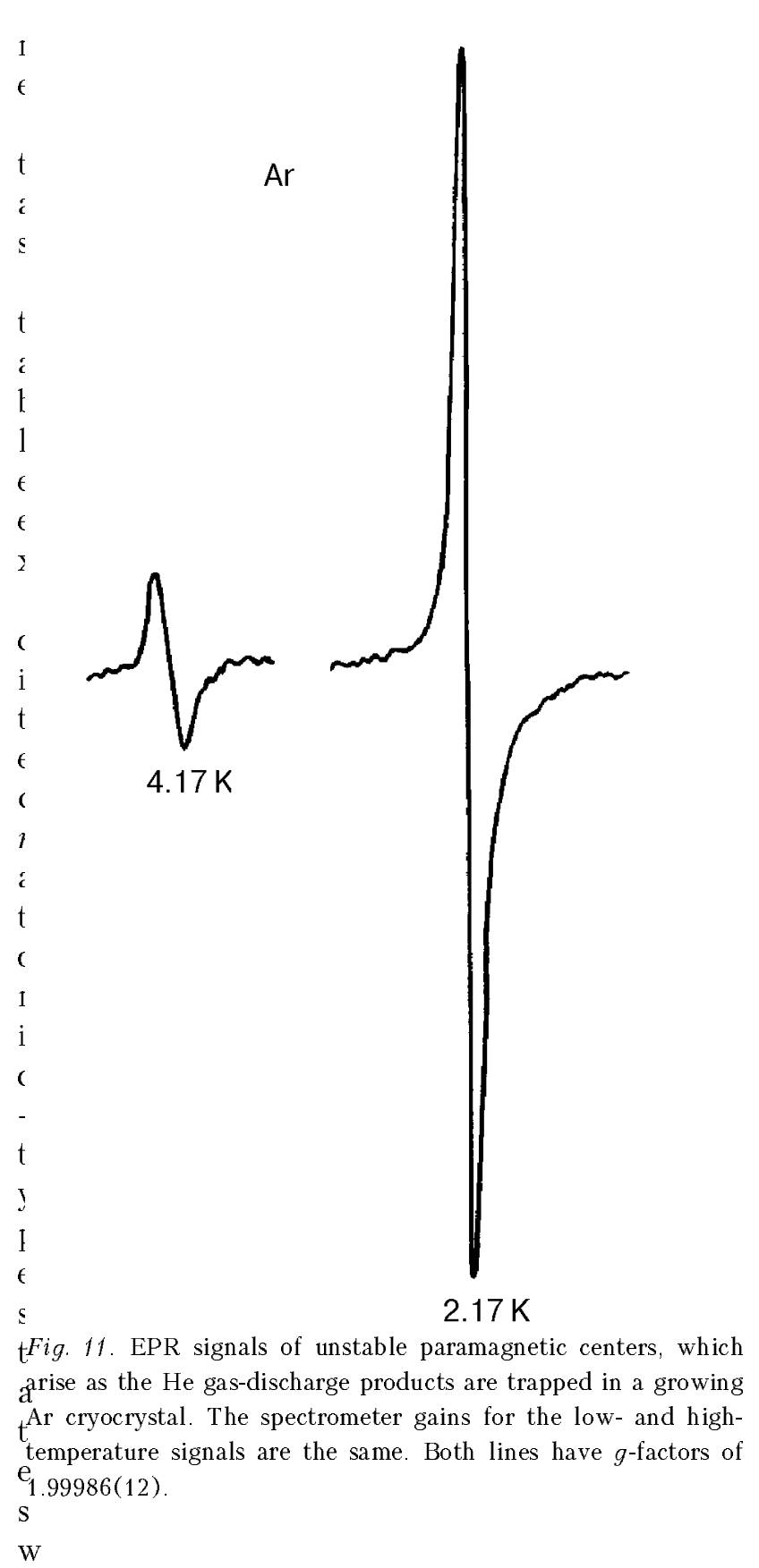

hich become localized in a face-centered cubic Ar $(\mathrm{Kr})$ crystal lattice, shift the nearest matrix atoms outwards, rearrange their surroundings and are subject to the action of the anisotropic electric field of this environment which, being rearranged, is no longer of cubic symmetry.

After the discovery in Ar and $\mathrm{Kr}$ cryocrystals of the EPR spectra presented in Figs. 11, 12 which appear as He gas discharge products are trapped in the growing cryocrystals, the dependence of the production probability of the local excited $n p^{5}(n+1) s$ atomic-type state in the cryocrystals on the sample temperature has been studied. Toward this goal, the signal intensity of the above spectra

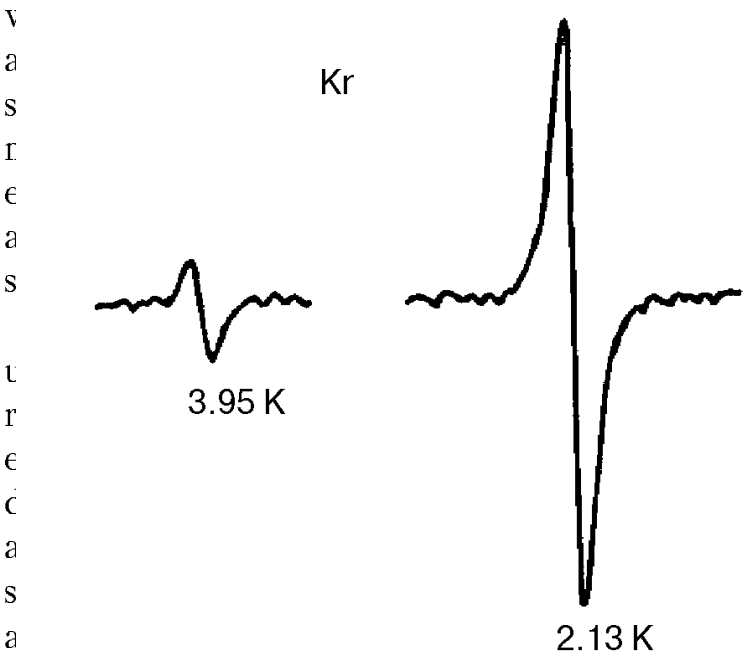

fFig. 12. EPR signals of unstable paramagnetic centers, which $\mathrm{u}^{\text {arise }}$ as the He gas-discharge products are trapped in a growing $\mathrm{Kr}$ cryocrystal. The spectrometer gains for the low- and hightemperature signals are the same. Both lines have $g$-factors of c.99986(12).

$\mathrm{t}$ ion of the substrate temperature.

Shown in Fig. 9 are the temperature dependences of the EPR line intensities that are typical of $\mathrm{Ar}$ (line 3) and $\mathrm{Kr}$ (line 4) cryocrystals. The EPR lines are like that depicted in Figs. 11, 12. The EPR signal intensities are measured in $A(T) / A\left(T_{0}\right)$ units, where, for the $\mathrm{Ar}$ and $\mathrm{Kr}$ cryocrystals, $T_{0}$ is the lowest temperature point at which the sample is still sufficiently stable. This gives the measure of the lowest possible temperature $T_{0}$ which somewhat changes from run to run $[11,12]$.

It is evident from Fig. 9 that the temperature dependence of the EPR signal intensity, i.e., the temperature dependence of the production probability of the atomic-type $n p^{5}(n+1) s$ excitations in $\mathrm{Ar}$ and $\mathrm{Kr}$ cryocrystals, can be well fitted by a linear function $A(T) / A\left(T_{0}\right)=-a\left(T-T_{0}\right)+b$. The experiments carried out show the line slope, or the magnitude of $a$, to vary within narrow limits comparable, presumably, to those of experimental error. A set of six experiments with Ar yields $a(\mathrm{Ar})$ changing from 0.33 to 0.44 , an average quantity being $\overline{a(\mathrm{Ar})}=0.39$, while three $\mathrm{Kr}$ runs give $a=$ $=0.31-0.57$ and $\overline{a(\mathrm{Kr})}=0.46$. In these experiments, $b$ ranges between $1.66-1.93$, for $\operatorname{Ar}(\overline{b(\mathrm{Ar})}=1.78)$, and between $1.82-2.38$, for $\operatorname{Kr}(\overline{b(\mathrm{Kr})}=2.11)$. If one considers the trapping of metastable $\mathrm{He} 2^{3} S_{1}$ atoms in neon cryocrystals (see Sec. 3), the temperature dependence has been shown to be exponential («Arrhenius-like») $A(T) / A\left(T_{1}\right)=\exp [-(E / k)(1 / T-$ - 1/ $\left.\left.T_{1}\right)\right]$, where $E=12(6) \mathrm{K}=0.0010(5) \mathrm{eV}$.

Thus, the temperature dependences of the $n p^{5}(n+1) s$ center production probability are closely 
related for the Ar and $\mathrm{Kr}$ cryocrystals while quite distinct from that of the Ne cryocrystal both in the trend and shape.

Examining experimental results outlined, the following mechanism of formation of the metastable $n p^{5}(n+1) s$ states in the growing $\mathrm{Ar}$ and $\mathrm{Kr}$ cryocrystals subjected to the action of He discharge products can be proposed.

There is no question the metastable He $2^{3} S_{1}$ and $2^{1} S_{0}$ atoms, in the present study, are trapped in great numbers in the growing $\mathrm{Ar}$ and $\mathrm{Kr}$ cryocrystals, for the trapping process goes on in these cryocrystals as it evidently does in Ne (Sec. 3.3). Excitation energies of the metastable $\mathrm{He}$ atoms $E\left(2^{3} S_{1}\right)=19.82 \mathrm{eV}$ and $E\left(2^{1} S_{0}\right)=20.62 \mathrm{eV}$ are considerably larger than the energy gaps of the Ar and Kr cryocrystals: $E_{g}(\mathrm{Ar})=14.16 \mathrm{eV}$ and $E_{g}(\mathrm{Kr})=$ $=11.61 \mathrm{eV}$ (Ref. 1, p. 27). Therefore, the trapped metastable He $2^{3} S_{1}$ and $2{ }^{1} S_{0}$ atoms transfer their excitation energies to the $\mathrm{Ar}$ or $\mathrm{Kr}$ cryocrystal to produce an internal ionization of this cryocrystal when a hole, i.e., $\mathrm{Ar}^{+}$or $\mathrm{Kr}^{+}$ion, arises in the valence band while a free electron $e$ appears in the conduction band. It is known [1] that in the rare gas cryocrystals the holes become self-trapped in a very short time $\left(10^{-12}-10^{-13} \mathrm{~s}\right)$ after their production to give rise to the molecular ions: $\mathrm{Rg}^{+}+\mathrm{Rg} \rightarrow \mathrm{Rg}_{2}^{+}$. Occupying first a vibrationally excited level, the molecular ion relaxes to the lowest vibrational levels transferring its energy to the phonon spectrum of the cryocrystal. At some time a free electron in the conduction band encounters a hole to recombine with it and produce an excited $\mathrm{Rg}_{2}^{* *}$ molecule which may, on occasion, dissociate, yielding an excited atom: $\mathrm{Rg}_{2}^{+}+e \rightarrow \mathrm{Rg}_{2}^{* *} \rightarrow \mathrm{Rg}^{*}+\mathrm{Rg}$. The process is known to occur both in the rare gases and in their cryocrystals [1]. This reaction of dissociative recombination may be one way to produce ultimately the metastable excited ${ }^{3} P_{2}$ atomic-type states in $\mathrm{Ar}$ and $\mathrm{Kr}$ cryocrystals observed in the present study $[11,12]$.

A plausible mechanism of production, in Ar and $\mathrm{Kr}$ cryocrystals, of the excited $n p^{5}(n+1) s$ states observed is discussed below in greater detail.

The process of formation of the $3 p^{5} 4 s$ states in $\mathrm{Ar}$ cryocrystals, having trapped the metastable $\mathrm{He}$ $2^{3} S_{1}$ and $2^{1} S_{0}$ atoms from the helium gas discharge, can be conceived of as follows $[11,12]$.

The trapped He $2^{3} S_{1}$ and $2^{1} S_{0}$ atoms transfer their excitation energies to the Ar cryocrystal, with the above-mentioned internal ionization of the cryocrystal, as a consequence, this results in the formation of a free electron in the conduction band and a hole $\mathrm{Ar}^{+}$in the valence band; the hole becomes rapidly self-trapped (in a time of $10^{-12}-10^{-13} \mathrm{~s}$ ), i.e., turns to the vibrationally excited molecular $\mathrm{Ar}_{2}^{+}$ion. Provided that, after a short time, a free electron moving in the conduction band encounters and recombines with an $\mathrm{Ar}_{2}^{+}$hole that has had no time to relax vibrationally, the reaction of dissociative recombination is possible: $\mathrm{Ar}_{2}^{+}+e \rightarrow \mathrm{Ar}_{2}^{* *} \rightarrow \mathrm{Ar}+\operatorname{Ar}^{*}\left(3 p^{5} 4 p\right)$. The reaction is followed by the excited atom decay $\operatorname{Ar}^{*}\left(3 p^{5} 4 p\right) \rightarrow \operatorname{Ar}^{*}\left(3 p^{5} 4 s\right)$ to form the $3 p^{5} 4 s^{3} P_{2}$ state observed by EPR. This is likely to be a mechanism of formation, in Ar cryocrystals, of the metastable excited $3 p^{5} 4 s$ atomic-type states as the excitation energy is being transferred from the metastable $\mathrm{He}$ atoms to the cryocrystal.

Suppose that the time a free electron moving in the conduction band takes to approach an $\mathrm{Ar}_{2}^{+}$ molecular ion is sufficiently long that the ion has relaxed to the lowest vibrational state. Then the energy released during the $\mathrm{Ar}_{2}^{+}+e$ recombination is allowed to transfer to the exciton band with $n=1$ $[11,12]$. This exciton then can either decay to the ground state or become self-trapped to the two-center excimer state, $\mathrm{Ar}_{2}^{*}$, which also decays to two ground-state $\mathrm{Ar}$ atoms. These processes cannot lead to the formation of the atomic-type $3 p^{5} 4 s$ state. That is why the process of production of the atomictype $3 p^{5} 4 s$ state through the recombination of the vibrationally relaxed $\mathrm{Ar}_{2}^{+}$molecular ion should be far less probable than the dissociative recombination of the vibrationally excited $\mathrm{Ar}_{2}^{+}$ion. Which of the two processes occurs in a particular experiment depends on the growing cryocrystal temperature. With increasing sample temperature the vibrational relaxation rate of the $\mathrm{Ar}_{2}^{+}$molecular ion rises, whereas the mobility of electrons in the conduction band grows smaller leading to an increase with sample temperature in the time separation between the $\mathrm{Ar}_{2}^{+}$ion production and its recombination with an electron. All these reasons have the effect of reducing the probability of the dissociative recombination and consequently the yield of $3 p^{5} 4 s$ centers, which underlies the temperature dependence found for Ar (Fig. 9). The probability of the excitation energy transfer to the exciton band with $n=1$ followed by decay to two ground-state Ar atoms increases with increasing sample temperature.

In the $\mathrm{Kr}$ cryocrystal, the processes must be somewhat different from those described above for Ar [11,12]. Metastable He atoms trapped in the growing $\mathrm{Kr}$ cryocrystal will also perform the internal ionization of the cryocrystal to produce in a short time $\left(10^{-12}-10^{-13} \mathrm{~s}\right)$ a vibrationally excited self-trapped hole, $\mathrm{Kr}_{2}^{+}$, and a free electron in the 
conduction band. At low temperatures when a molecular ion $\mathrm{Kr}_{2}^{+}$recombining with an electron should be vibrationally excited, the most likely mechanism of the energy loss is that through the excitation energy transfer to the exciton band with $n=1$, $\Gamma(1 / 2)$ followed by the self-trapping to the $4 p^{5} 5 s$ state and production of the excited ${ }^{3} P_{2}$ state detected in the experiment by EPR. The energy gap between $\Gamma(1 / 2)$ and $\Gamma(3 / 2)$ subbands of the exciton with $n=1$ must hamper non-radiative transitions from the first subband to second in the process $[11,12]$. It seems possible that the dissociative recombination, $\mathrm{Kr}_{2}^{+}+e \rightarrow \mathrm{Kr}_{2}^{* *} \rightarrow \mathrm{Kr}+\mathrm{Kr}^{*}\left(4 p^{5} 5 s\right)$, can also contribute to the observed signal, giving metastable $4 p^{5} 5 s$ state. As the sample temperature increases, the vibrational relaxation of the $\mathrm{Kr}_{2}^{+}$ molecular ion speeds up while the movement of electrons in the conduction band slows down. This brings about the recombination, $\mathrm{Kr}_{2}^{+}+e$, in which case the excitation energy is likely to be transferred to the exciton band with $n=1, \Gamma(3 / 2)$ with subsequent decay to the ground state either immediately from the subband with $n=1, \Gamma(3 / 2)$ or through the self-trapped excimer $\mathrm{Kr}_{2}^{+}$state. This will lead to a decrease in the yield of the metastable excited ${ }^{3} P_{2}$ states as the cryocrystal temperature increases. The processes discussed above are responsible for the fall in the $4 p^{5} 5 s$ center yield in the $\mathrm{Kr}$ cryocrystal with increasing temperature. It is of interest that the temperature dependences of the probability of the $n p^{5}(n+1) s$ center formation for $\mathrm{Ar}$ and $\mathrm{Kr}$ cryocrystals are similar in appearance (Fig. 9), notwithstanding certain distinctions between mechanisms of production of these metastable atomic-type centers.

Thus, the metastable excited He atoms, He $\left(2^{3} S_{1}\right)$ and $\mathrm{He}\left(2^{1} S_{0}\right)$, from the He gas discharge become trapped in the growing $\mathrm{Ar}$ or $\mathrm{Kr}$ cryocrystals and transfer their excitation energy to the cryocrystal to form, in the process of internal ionization, $\mathrm{a} \mathrm{Rg}^{+}$ion and a free electron in the conduction band, whereupon the fast (of $10^{-12} \mathrm{~s}$ ) self-trapping reaction of a hole follows: $\mathrm{Rg}^{+}+\mathrm{Rg} \rightarrow \mathrm{Rg}_{2}^{+}$. Thereafter either the dissociative recombination reaction $\mathrm{Rg}_{2}^{+}+e \rightarrow$ $\rightarrow \mathrm{Rg}_{2}^{* *} \rightarrow \operatorname{Rg}+\operatorname{Rg}\left[n p^{5}(n+1) s\right]$ or recombination $\mathrm{Rg}_{2}^{+}+e \rightarrow \mathrm{Rg}+\mathrm{Rg}$ to produce ground-state atoms could take place. The former is likely at lower temperatures while the latter - at higher temperatures when the vibrational relaxation rate of the $\mathrm{Rg}^{+}$molecular ion increases and the mobility of free electrons in the conduction band decreases. This is the reason for the observed temperature dependence $[11,12]$.

\subsection{Additional check experiments}

This section is on examining the possibility of the unstable centers in rare gas cryocrystals observed and discussed in Refs. 7-12 can be due to any atomic or molecular paramagnetic impurity ions matrix isolated in the cryocrystals. Uncharged impurity atoms and molecules should be excluded at once from consideration, since they are known to give only stable paramagnetic centers in the matrices of solid rare gases, as was shown in many works (for example, on $\mathrm{H}, \mathrm{D}$ and $\mathrm{N}$ atoms $[19,20]$ ). At the same time, matrix-isolated ions may in principle give rise to unstable paramagnetic centers with limited lifetime $\tau$, since the ions may be suggested to recombine, after a time lapse of $\tau$, with free electrons existing in the matrix to form uncharged diamagnetic particles. However, both ions and electrons were demonstrated above to be unable, in the described experiments, to travel from the gas discharge onto the growing cryocrystal, because they are locked in the discharge zone with the magnetic field of the EPR spectrometer $H=3300 \mathrm{G}$ forcing them to follow small-radius trajectories (thus, for $\mathrm{O}^{+}$ion the orbit radius is under $0.1 \mathrm{~mm}$ ).

At the same time, uncharged atoms and molecules presented as natural impurities in the rare gas discharge can reach the substrate and trap within the growing cryocrystal. Thus, the processes may be imagined to occur as follows: uncharged impurity atoms or molecules passing onto the growing cryocrystal are subject to ionization, near the surface or in the near-surface layer, by VUV radiation from the gas discharge, whereupon these ions, along with electrons, are trapped in the cryocrystal to give rise to the observed EPR spectra following which the recombination, after a time lapse of $\tau$, of the ions with trapped electrons takes place with the result that uncharged diamagnetic particles appear, this might provide a nonstability of the observed EPR spectra. The present section is concerned with experiments examining if the above mechanism might be responsible for the EPR unstable signals observed in the rare gas cryocrystals.

It should be noted first that if the matrix-isolated paramagnetic ions which after a certain time become uncharged and diamagnetic through recombination with electrons make the unstable centers observed, than the EPR signals they yield cannot disappear completely once the discharge is turned off but at least a portion of signal must remain and be stable. The reason is that a rare gas cryocrystal being condensed contains enough abundance of electron traps like impurity molecules and atoms, and lattice defects. These traps bind electrons to inhibit 
them from recombining with ions, so the later become stable matrix-isolated paramagnetic particles in the cryocrystal and yield stable EPR spectra. However, in all experiments, all lines of unstable paramagnetic EPR spectra under study disappear completely after the discharge is turned off. This testifies against the hypotheses about the impurity paramagnetic ions.

As a finish check on the suggestion that unstable EPR spectra observed are due to impurity paramagnetic ions specialized experiments were run. In these experiments, the growing rare gas cryocrystal which trapped gas discharge products was added with great deal of effective electron traps which were to be result in large numbers of completely stable ions and thereby to stabilize the observed signals which had been quite unstable until then. As an acceptor to create effective electron traps, the oxygen was chosen because of the great electron affinity of both the molecule and the atom: $E_{a}\left(\mathrm{O}_{2}\right)=0.440(8) \mathrm{eV}, E_{a}(\mathrm{O})=1.465 \mathrm{eV}$ [21]. A deposited cryocrystal of a rare gas has been demonstrated [22] to contain practically always a sufficient number of electron traps, the natural $\mathrm{O}_{2}$ impurity (in small amounts) among them, to stabilize completely a variety of matrix-isolated ions. However, in certain cases [23] an amount of $\mathrm{Cl}_{2}$ with the electron affinity of $E_{a}\left(\mathrm{Cl}_{2}\right)=2.38 \mathrm{eV}$ was added to the matrix as an electron acceptor to enhance the number of stabilized ions in the rare gas cryocrystals. In the first stage of the check study being discussed in the present section, gaseous $\mathrm{O}_{2}$ was added to the pure $\mathrm{Ne}$ or $\mathrm{Ar}$ passed through the channel $A$ (channel $B$ was shut). The EPR unstable signals like that depicted in Fig. 2, which are characteristic for the pure $\mathrm{Ne}$ and $\mathrm{Ar}$, were detected; these signals disappeared completely once the gas discharge was turned off, i.e., no evidence of stable EPR spectra was observed. The addition of oxygen in the gas discharge resulted only in decreased signal amplitude. Based on the interpretation discussed in Sec. 3.3, this could be explained by the de-excitation of the rare gas ${ }^{3} P_{2}$ atoms at collisions with $\mathrm{O}_{2}$ molecules in the gas discharge, which would tend to the Penning ionization of the molecules. It should be noted that $\mathrm{O}^{+}$ions are particularly promising when trying to provide an explanation for the observed unstable signals as being matrix-isolated paramagnetic ions which subsequently recombine with electrons. First, $\mathrm{O}$ atoms, like the stable matrix-isolated $\mathrm{N}$ atoms which characteristically are easy to observe in the EPR spectra, are undoubtedly present in the gas discharge and, to be sure, are also trapped and matrix-isolated in the cryocrystal (though the observation of their EPR spectra have not been successful). Yet, only the Ne gas discharge provides VUV photons with the energy high enough $(16.8 \mathrm{eV})$ to ionize oxygen atoms $\left(E_{i}=13.6 \mathrm{eV}\right)$ near the cryocrystal surface while, in the case of $\mathrm{Ar}, \mathrm{Kr}$ and $\mathrm{Xe}$, the gas discharge produces photons of too low energy (11.7, eV, $10.6 \mathrm{eV}$ and $9.6 \mathrm{eV}$, respectively) to do this. Second, $\mathrm{O}^{+}$ion is promising again in this consideration because of its $2 s^{2} 2 p^{3}{ }^{4} S_{3 / 2}$ state, as is the case for the nitrogen atom also, which provides $g=2.0$ for the free $\mathrm{O}^{+}$ion even without the action of the crystal field. However, in the considered experiments, the addition of $\mathrm{O}_{2}$ to the gas discharge did not resulted in both stabilization and rise of the observed spectra in $\mathrm{Ne}$ and $\mathrm{Kr}$.

In subsequent experiments, the gaseous $\mathrm{O}_{2}$ was added through the channel $B$ (Fig. 1) so that it had no action on the gas discharge, whereas only pure rare gas (Ne or Ar) was passed through the channel $A$ and gas discharge. Shown in Fig. 13 is the amplitude of the pure Ne central line (Fig. 2) plotted against the $\mathrm{O}_{2}$ pressure in the channel $B$ (Fig. 1). From this figure it is noticed that here, too, the unstable signals show no increase in amplitude on addition of oxygen into the growing Ne cryocrystal, bypassing the gas discharge, as might be hoped for, but a rapid decline. This influence of $\mathrm{O}_{2}$ is fully reversible - as the oxygen flow rate through the channel $B$ decreases, the EPR signal grows in amplitude, following the same curve as in Fig. 13, and, thus, regains its initial value completely as the oxygen flow comes to an end.

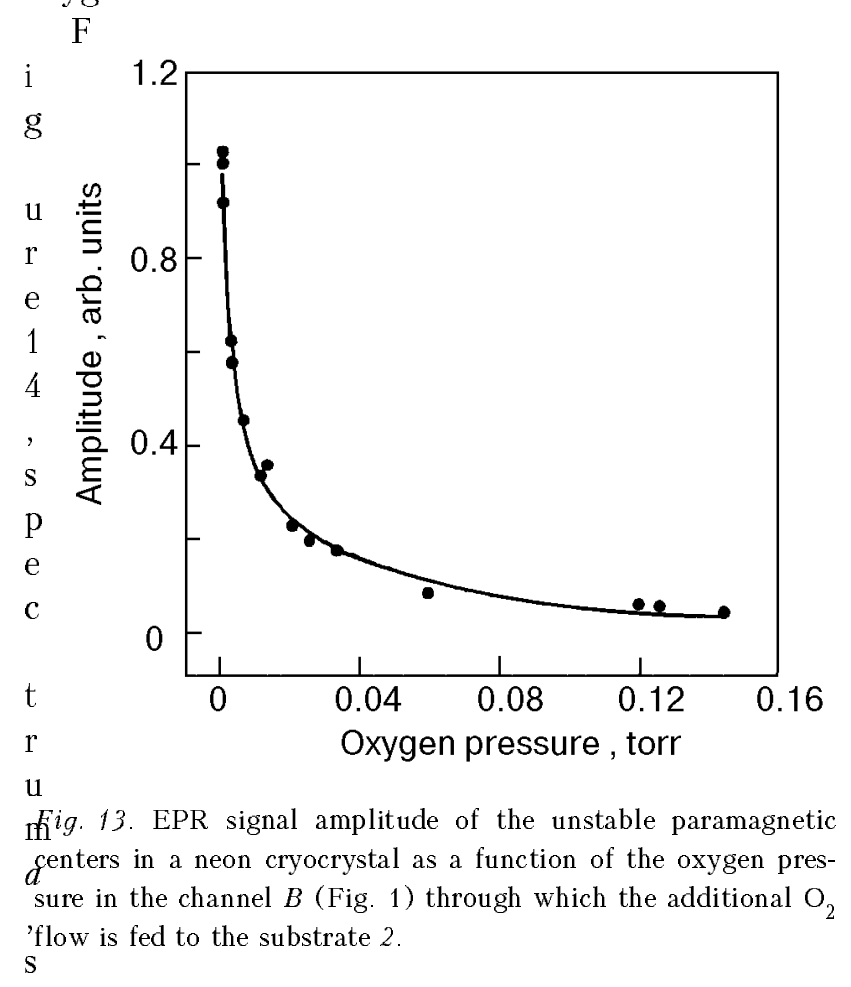




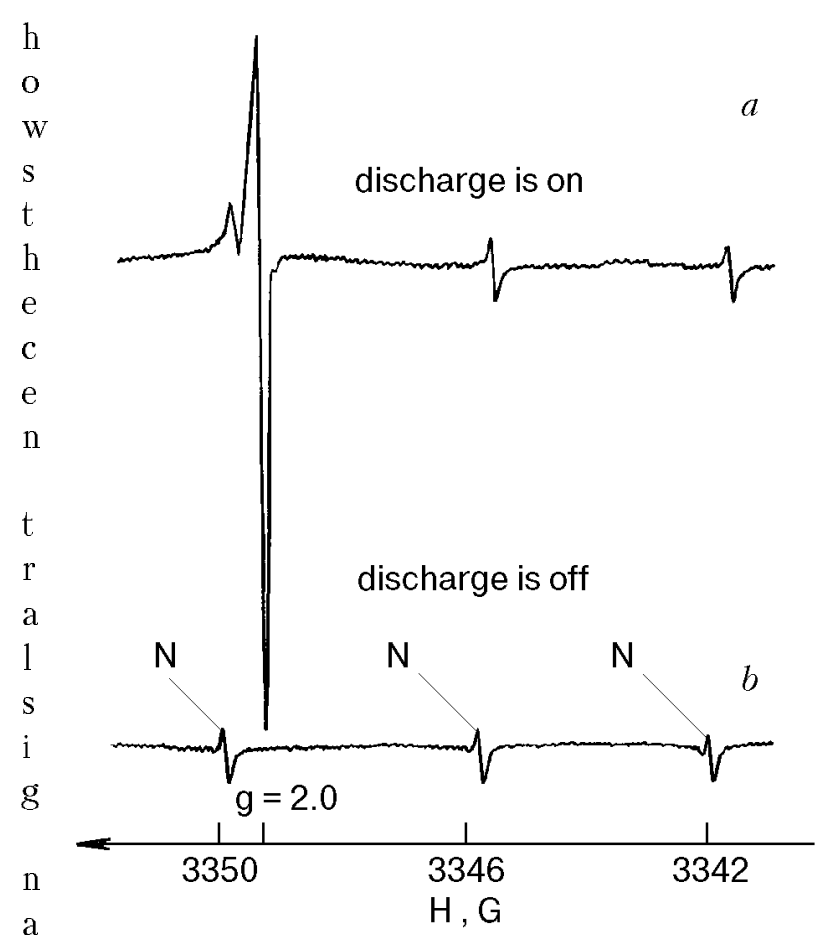

Fig. 14. EPR spectra of a neon cryocrystal recorded after a four-hours run in which the gaseous neon was passed through

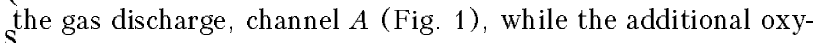
gen was fed through the channel $B$ with the flow rate changing during the run. Record $a-$ EPR spectrum taken as the neon discharge in the channel $A$ runs and the oxygen flow in Fthe channel $B$ is cut off; record $b-$ EPR spectrum recorded ijust after the spectrum $a$ was taken and the discharge in changel $A$ turned off. The unstable signal with $g=2.0$ disappears completely, while the week signals of $\mathrm{N}$-atoms trapped in the $2^{\text {neon }}$ cryocrystal remain unchanged, which relates to the well known stability of the spectrum of nitrogen atoms matrix-isolated in $\mathrm{Ne}$.

$\mathrm{t}$

he oxygen flow being cut off, recorded after a four-hours run in which the oxygen has been introduced into the growing neon cryocrystal through the channel $B$ and dependences like that depicted in Fig. 13 have been taken. As this took place, the maximum $\mathrm{O}_{2}$ flow rate onto the substrate 2 through the channel $B$ was something of an order of magnitude greater than the flow rate onto the substrate of the rare gas through the channel $A$. The signal amplitude in Fig. 14, spectrum $a$, returned to that of the pure $\mathrm{Ne}$ at the onset of experiment, before $\mathrm{O}_{2}$ was let in. Also seen in Fig. 14, spectrum $a$, are three lines of the $\mathrm{N}\left({ }^{4} S_{3 / 2}\right)$ spectrum which are due to uncontrollable small amounts of nitrogen in the gas discharge. Such spectra of the N-atoms matrixisolated in a neon cryocrystal have been observed earlier [20]. The spectrum $b$ in Fig. 14 was taken just after the discharge was turned off. It is evident from the figure that the signal like that depicted in Fig. 2 disappears completely after the discharge is turned off, i.e., remains fully unstable after such a prolonged experiment, in spite of great quantity of effective electron traps such as $\mathrm{O}_{2}$ molecules in the cryocrystal. This permits one to rule out totally the suggestion that the unstable paramagnetic centers which were observed in rare gas cryocrystals present matrix-isolated ions, whatever they may be. In Fig. 14, spectrum $b$, the lines of the matrix-isolated $\mathrm{N}$ atoms which are kept constant testifies to reliability of this inference.

Mention may be made that, in the present study, the possibility of the observed spectra owing to matrix-isolated positive ions (cations) is investigated. With negative ions (anions), they should, once matrix-isolated, yield stable EPR signals much as uncharged paramagnetic particles do, since these fixed negative ions cannot recombine in a low temperature matrix.

\section{Conclusion}

Thus, the arguments of Sec. 3.2, the addition check experiments wherein an electron acceptor $\mathrm{O}_{2}$ was admitted, and the temperature investigations (Fig. 9) essentially exclude all sorts of alternative explanations of the unstable paramagnetic centers observed in the rare gas cyocrystals $\mathrm{Ne}, \mathrm{Ar}, \mathrm{Kr}$, and $\mathrm{Xe}$ that are subject to action of the products of gas discharge in either these gases or He trapped in the growing cryocrystal. Consequently, it is proved that the unstable paramagnetic centers observed by EPR present the metastable excited $n p^{5}(n+1) s$ atomic-type states which are localized in the cryocrystal, deform and rearrange their nearest surroundings, and are subject to the action of the anisotropic electric field caused by these deformed surroundings.

Figure 9 (lines 1 and 2), shows a surprising phenomenon: completely dissimilar temperature dependences for the equilibrium concentration of the same centers in the same substance. The only explanation is that the unstable centers of the same nature and in the same substance are in fact formed in various ways. The reasonable and compatible explanation for this complicated effect which is given above (Secs. 3.2, 3.3, and 3.4) is a weighty argument in support of correctness of this explanation. Distinction between temperature dependences for the same centers allows one to exclude also the possibility that they are some radiation-induced paramagnetic centers formed in the quartz substrate under the action of the gas discharge radiation. In that case, one could not explain the observed distinction between temperature dependences. The fact that the centers disappear once the $\mathrm{Ne}, \mathrm{Ar}$ or $\mathrm{Kr}$ 
flow through the channel $B$ is cut off (see Secs. 3.3 and 3.4), whereas the substrate is still exposed to the He gas discharge radiation, is also a strong evidence against this suggestion.

Special experiments were also carried out, using the set-up depicted in Fig. 1, to try to discover unstable EPR spectra close to $g=2$ (like that described above) in pure $\mathrm{H}_{2}$ and $\mathrm{N}_{2}$ cryocrystals trapping products of the gas discharge in the same gases, $\mathrm{H}_{2}$ or $\mathrm{N}_{2}$. No unstable EPR spectra close to $g=2$ were detected in these pure cryocrystals in the runs. This is a further evidence for the above interpretation of the nature of the unstable paramagnetic centers in the rare gas cryocrystals which were observed.

1. N. Schwentner, E. E. Koch, and J. Jortner, Electronic Excitations in Condensed Rare Gases, Springer-Verlag, Berlin-Heidelberg (1985).

2. M. Ueta, H. Kanzaki, K. Kobayashi, Y. Toyozawa, and E. Hanamura, Excitonic Processes in Solids, Springer-Verlag, Berlin-Heidelberg (1986).

3. K. S. Song and R. T. Williams, Self-Trapped Excitons, Springer-Verlag, Berlin-Heidelberg (1996).

4. E. I. Rashba, in: Excitons, E. I. Rashba and M. D. Struge (eds.), North-Holland, Amsterdam (1982), p. 543.

5. E. V. Savchenko and I. Ya. Fugol', in: Cryocrystals, B. I. Verkin and A. F. Prihot'ko (eds.), Naukova Dumka, Kiev (1983) (in Russian), p. 360.

6. G. Zimmerer, in: Excited State Spectroscopy in Solids, U. M. Grassano and N. Terzi (eds.), North-Holland, Amsterdam (1987), p. 37.

7. R. A. Zhitnikov, Yu. A. Dmitriev, and M. E. Kaimakov, Sov. Phys. JETP 22, 1009 (1991).
8. R. A. Zhitnikov, Yu. A. Dmitriev, and M. E. Kaimakov, Sov. J. Low Temp. Phys. 18, 532 (1992).

9. R. A. Zhitnikov and Yu. A. Dmitriev, Sov. J. Low Temp. Phys. 19, 437 (1993).

10. R. A. Zhitnikov and Yu. A. Dmitriev, J. Phys.: Condens. Matter 6, 2727 (1994).

11. R. A. Zhitnikov and Yu. A. Dmitriev, J. Phys.: Condens. Matter 7, 2819 (1995).

12. R. A. Zhitnikov and Yu. A. Dmitriev, Appl. Magn. Reson. 8, 457 (1995).

13. A. A. Radtsig and B. M. Smirnov, The Parameters of Atoms and Atomic Ions, Energoatomizdat, Moskow (1986) (in Russian).

14. T. Suemoto and H. Kanzaki, J. Phys. Soc. Jpn. 46, 1554 (1979).

15. J. E. Wertz and J. R. Bolton, Electron Spin Resonance. Elementary theory and Practical Applications, McGrawHill, New York (1972).

16 F. V. Kusmartsev and E. I. Rashba, Czech. J. Phys. B32, 54 (1982)

17. Chun-rang Fu and K. S. Song, J. Phys.: Condens. Matter 9, 9785 (1997)

18. F. V. Kusmartsev and E. I. Rashba, Sov. Phys. JETP 59, 668 (1984).

19. R. A. Zhitnikov and Yu. A. Dmitriev, Sov. Phys. JETP 65, 1075 (1987).

20. Yu. A. Dmitriev and R. A. Zhitnikov, Sov. Phys. Tech. Phys. 32, 1082 (1987).

21. A. A. Radtsig and B. M. Smirnov, Atomic and Molecular Physics Handbook, Atomizdat, Moskow (1980) (in Russian).

22. L. B. Knight, Jr. and J. Steadman, J. Chem. Phys. 77, 1750 (1982).

23. L. B. Knight, Jr. and J. Steadman, J. Chem. Phys. 78, 5940 (1983). 